\title{
CircRNA inhibits DNA damage repair by interacting with host gene
}

Xiaolong Xu ${ }^{1 \dagger}$, Jingwei Zhang ${ }^{2 \dagger}$, Yihao Tian ${ }^{1 \dagger}$, Yang Gao ${ }^{1 \dagger}$, Xin Dong ${ }^{1}$, Wenbo Chen ${ }^{1}$, Xiaoning Yuan ${ }^{1}$, Weinan Yin ${ }^{1}$, Jinjing $\mathrm{Xu}^{2}$, Ke Chen ${ }^{3^{*}}$, Chunjiang $\mathrm{He}^{1,4^{*}}$ (D) and Lei Wei ${ }^{1,5^{*}}$

\begin{abstract}
Background: Deregulated circular RNAs (circRNAs) are associated with the development of cancer and therapy resistance. However, functional research of circRNAs mostly focus on potential miRNA or protein binding and more potential regulation of circRNA on host gene DNA in cancers are yet to be inspected.

Method: We performed total RNA sequencing on clinical breast cancer samples and identified the expression patterns of circRNAs and corresponding host genes in patient blood, tumor and adjacent normal tissues. qPCR, northern blot and in situ hybridization were used to validate the dysregulation of circRNA circSMARCA5. A series of procedures including R-loop dot-blotting, DNA-RNA immunoprecipitation and mass spectrum, etc. were conducted to explore the regulation of circSMARCA5 on the transcription of exon 15 of SMARCA5. Moreover, immunofluorescence and in vivo experiments were executed to investigate the overexpression of circSMARCA5 with drug sensitivities.
\end{abstract}

Results: We found that circRNAs has average higher expression over its host linear genes in peripheral blood. Compared to adjacent normal tissues, circSMARCA5 is decreased in breast cancer tissues, contrary to host gene SMARCA5. The enforced expression of circSMARCA5 induced drug sensitivity of breast cancer cell lines in vitro and in vivo. Furthermore, we demonstrated that circSMARCA5 can bind to its parent gene locus, forming an R-loop, which results in transcriptional pausing at exon 15 of SMARCA5. CircSMARCA5 expression resulted in the downregulation of SMARCA5 and the production of a truncated nonfunctional protein, and the overexpression of circSMARCA5 was sufficient to improve sensitivity to cytotoxic drugs.

Conclusion: Our results revealed a new regulatory mechanism for circRNA on its host gene and provided evidence that circSMARCA5 may serve as a therapeutic target for drug-resistant breast cancer patients.

Keywords: Breast cancer, circRNA, DNA damage repair, R-loop, Host gene

\footnotetext{
*Correspondence: kechen@hust.edu.cn; che@whu.edu.cn; leiweifr@hotmail.com

${ }^{+}$Xiaolong Xu, Jingwei Zhang, Yihao Tian and Yang Gao contributed equally to this work.

${ }^{3}$ Department of Urology, Tongji Hospital, Tongji Medical College, Huazhong University of Science and Technology, Wuhan 430030, China

${ }^{1}$ School of Basic Medical Sciences, Wuhan University, Wuhan 430071, Hubei, China

Full list of author information is available at the end of the article
}

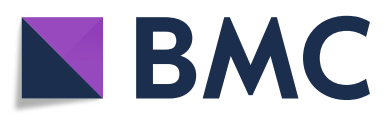

(- The Author(s). 2020 Open Access This article is licensed under a Creative Commons Attribution 4.0 International License, which permits use, sharing, adaptation, distribution and reproduction in any medium or format, as long as you give appropriate credit to the original author(s) and the source, provide a link to the Creative Commons licence, and indicate if changes were made. The images or other third party material in this article are included in the article's Creative Commons licence, unless indicated otherwise in a credit line to the material. If material is not included in the article's Creative Commons licence and your intended use is not permitted by statutory regulation or exceeds the permitted use, you will need to obtain permission directly from the copyright holder. To view a copy of this licence, visit http://creativecommons.org/licenses/by/4.0/ The Creative Commons Public Domain Dedication waiver (http://creativecommons.org/publicdomain/zero/1.0/) applies to the data made available in this article, unless otherwise stated in a credit line to the data. 


\section{Introduction}

Circular RNAs (circRNAs) are novel RNAs that have been ubiquitously discovered in many species by highthroughput sequencing in recent years $[1,2]$. CircRNAs are generated by the back-splicing of intronic, exonic or intergenic regions. circRNAs are resistant to RNase R, and the stability of their structures makes these molecules ideal candidates for disease [3]. Extensive studies have revealed that dysregulated circRNAs are involved in the development of various cancers. In gastric cancer, circRNAs, such as circPVT1, circLARP4, has_circ_ 0000096, and circ_100269, have been shown to play a role in promoting tumor growth, and their expression is correlated with high TNM stage and poor prognosis [4-7]. In colon and hepatic carcinoma, ciRS-7 promoted tumor development and progression by activating the EGFR and PI3K/Akt pathway [8, 9]. CircRNAs, such as circKIF4A, hsa_circ_0001944, hsa_circ_0001481 and circRNA_0025202, have been implicated in molecular typing, brain metastasis and drug resistance in breast cancer [10-12]. Although great progress has been made, the roles of circRNA and relevant molecular mechanisms remain largely unknown.

Previous studies have shown that circRNAs exert their functions in different ways. As noncoding RNAs, circRNAs regulate the expression of other genes by serving as sponges for microRNA and RNA-binding proteins $[13,14]$. In addition, some circRNAs have been shown to be translated into functional proteins $[15,16]$. In addition, circRNAs have also been shown to directly interact with the genomic DNA of the host gene in plant, which results in altered parent gene expression [17]. However, the interaction of circRNAs and host gene DNA were less studied in human cancers.

SMARCA5 is a member of the SWI/SNF complex with ATP-dependent chromatin remodeling activity [18-20]. In the process of DNA damage repair, SMARCA5 is involved in chromatin remodeling in DNA damage regions, providing a structural basis for the recruitment of DNA damage repair factors [21, 22]. In tumors, SMARCA5 is highly expressed in hepatic carcinoma and prostate cancer, and its expression level is inversely related to tumor radiosensitivity $[23,24]$.

In this study, we established circRNAs have average higher expression than their host genes in peripheral blood, comparing to tissues. Then we identified a circRNA derived from SMARCA5 (circSMARCA5) is significantly decreased in breast cancer cell lines and breast cancer samples. Different to previous works revealing circSMARCA5 can also function as a competing endogenous RNAs by binding with miRNA molecules [25-28], our mechanism exploration displayed circSMARCA5 is involved in regulating DNA repair capacity by binding exon DNA directly. And further functional investigation of this circRNA may contribute to the therapeutic implications for cytotoxic drug-resistant breast cancer patients.

\section{Results}

Identification of expression of circRNAs in breast cancer

We performed high throughput sequencing on tumor (T) and adjacent normal tissue (AN) and peripheral blood (B) of six breast cancer patients. Total RNA with rRNA-depleted library were constructed and then circRNAs expressed in those samples were identified. Compared to tumor and adjacent normal tissue, we observed average higher CIRCscore (expression of circRNA / linear host genes) in blood than both tumor and adjacent normal tissue. In all 8312 circRNAs which were expressed across all six patients, we observed average CIRCscores from 0.23 to 1.28 in blood, which is higher than tumor (0.05 to 0.11 ) and adjacent normal tissue (0.08 to 0.18$)$ in six patients (Fig. 1a). This result indicated average higher expression of circRNAs than their host genes in peripheral blood, comparing to tissues, which might contribute to the exploration of diagnostic biomarker for breast cancer. We then selected six circRNAs with high CIRCscores (average 0.22 to 15.8 in 6 patients) and performed further experimental validation in 24 patients. Real-time PCR results established two circRNAs (circHIPK3 and circSMARCA5) were significantly differentially expressed between tumor and adjacent normal tissue (Fig. $1 \mathrm{~b}$ and Figure S1A). Especially, circSMARCA5 was lower expressed in tumor samples and less studied in previous work. Furthermore, the ratio of circ-to-linear (expression of circRNA / linear host genes) of circSMARCA5 in blood sample of 24 health volunteers were significantly higher than those of 24 breast cancer patients $(P<0.05)$ (Fig. 1c and Figure S1B). We next examined the ratio of circ-to-linear of circSMARCA5 and clinical relevance in patients with breast cancer and observed significant difference in the distribution of the patients according to pathologic $\mathrm{T}(P=$ 0.038) (Table S1). Together, these results indicating the potential function and candidate biomarker attributes of circSMARCA5 in breast cancer.

To characterize and functionally investigate cirCSMARCA5, we firstly detected the expression of circSMARCA5 in cell lines. circSMARCA5 is derived from the back-splicing of exon 15 and exon 16 of SMARCA5 (Fig. 1d). As expected, endogenous circSMARCA5, but not pre-mRNA, was resistant to RNase $\mathrm{R}$ digestion (Fig. 1d). In addition, the existence of the $269 \mathrm{nt}$ circSMARCA 5 was further confirmed by Northern blot assay (Fig. 1e). Furthermore, we found that circSMARCA5 was mainly present in the nucleus, whereas its parent mRNA was present exclusively in the cytoplasm, as evidenced by qPCR, Northern blotting and RNA in situ hybridization (Fig. 1f-h and Figure S2). 


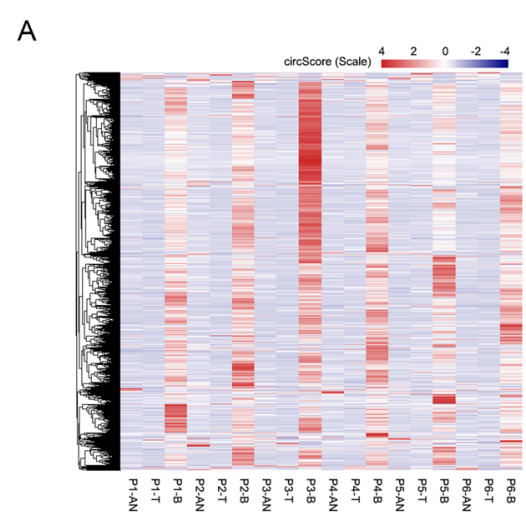

B

C

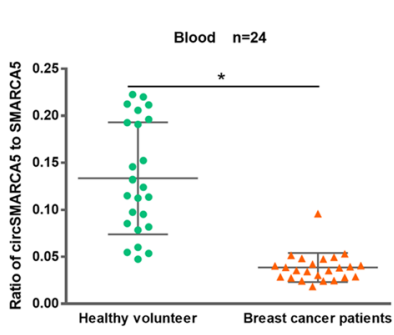

E
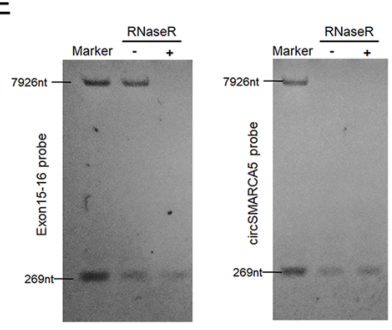

F

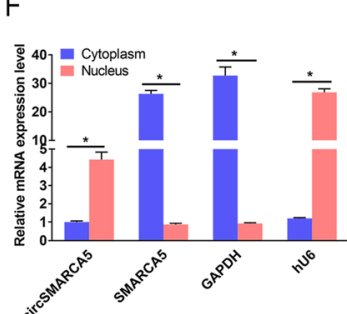

G

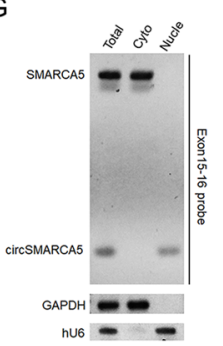

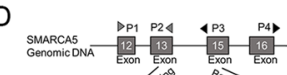
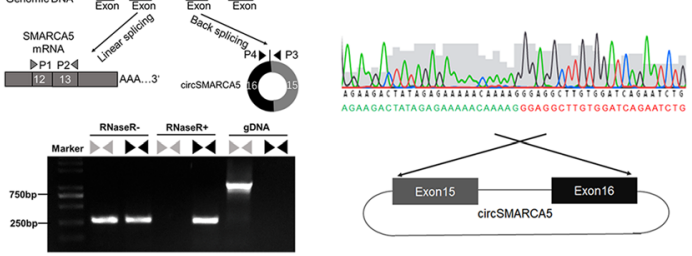

nus - -

$\mathrm{H}$
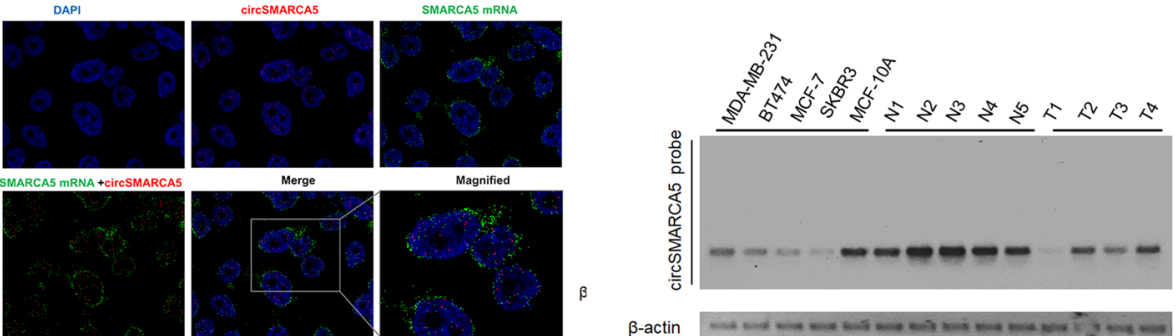

Fig. 1 (See legend on next page.) 


\begin{abstract}
(See figure on previous page.)
Fig. 1 Identification of circRNAs in breast cancer. a Heatmap of CIRCscore (FBPcirc/FBPlinear) in tumor (T), adjacent normal tissue (AN) and blood sample (B) from six breast cancer patients. $\mathbf{b}$ Expression of six circRNAs with high CIRCscore were validated by RT-qPCR assay in breast tumor and adjacent normal tissue. ${ }^{* *}$ represents $P<0.01$. CircRNAs IDs are according to circBase through their genomic coordinates. $\mathbf{c}$ The ratio of circ-to-linear of circSMARCA5 in blood sample of breast cancer patients and health volunteers. Total RNA from blood sample of breast cancer patients and health volunteers was extracted and detected by RT-qPCR. The expression level was normalized with $\beta$-actin as reference. *: $P<0.05$ was considered statistically significant. $\mathbf{d}$ Schematic illustration showing the genomic region of circSMARCA5 derived from exons 15 and 16 of the SMARCA5 gene. Convergent (gray) and divergent (black) primers were designed to amplify the linear or back-splicing products (upper). Total RNA from MCF-7 cells with or without RNase R treatment was subjected to RT-PCR (lower) and further validated by Sanger sequencing (Right). e Northern blot using a junction-specific probe or an exon 15-16 probe showing the endogenous existence of circSMARCA5 and SMARCA5 mRNA from MCF-7 cells with or without RNase-R treatment (R+ or R-). The 7926 bp marker indicates the SMARCA5 full-length transcript transcribed in vitro. The 269 bp marker indicates exon 15 and exon 16 of SMARCA5 transcribed in vitro. f The nucleus and cytoplasm mRNA of MCF-7 were extracted, and SMARCA5 and circSMARCA5 expression levels were quantitated by RT-PCR. GAPDH and hU6 serve as internal references of the cytoplasm and nucleus, respectively. "***" indicates $P<0.01$. g The nucleus and cytoplasm mRNA of MCF-7 were extracted, SMARCA5 and circSMARCA5 were examined by Northern blotting, and the SMARCA5 exon 15-16 probe was applied in this experiment. $\mathbf{h}$ Subcellular localization of circSMARCA5 and SMARCA5 in MCF-7 cells. The signals were examined by indirect RNA FISH and confocal microscopy. The nucleus was counterstained with DAPI. The circSMARCA5 probe was labled by biotin, while the SMARCA5 probe was labled by DIG. They were stained with red and green fluorescent secondary antibodies, respectively (I) The expression of circSMARCA5 detected by northern blot. MDA-MB-231, BT474, MCF-7, SKBR3 are breast cancer cell lines. MCF-10A are normal breast cell line. N1,N2,N3,N4,N5 are adjacent normal tissues. T1,T2,T3,T4 are breast cancer tissues. "***" indicates $P<0.01$
\end{abstract}

Next, we examined the expression of circSMARCA5 in various breast cancer cell lines (MCF7, SKBR3, BT474, MDA-MB-231) and immortalized but nontransformed breast epithelial cells (MCF-10A) as well as in adjacent normal tissues and breast cancer tissues. Northern blot results revealed that the expression levels of circSMARCA5 in MCF-10A and normal adjacent tissues are higher than breast cancer cell lines and cancer tissues (Fig. 1i). These results indicated that circSMARCA5 is downregulated in breast cancer tissues and cells.

\section{circSMARCA5 decreases the expression of SMARCA5 in cancer cells}

To clarify the mechanisms of circSMARCA5, we investigated its effects on the expression of its parent gene SMARCA5. The expression levels of circSMARCA5 and SMARCA5 were detected by the primers of junction sequence and 22-23 exons sequence, respectively. Knockdown of circSMARCA5 increased both mRNA and protein levels of SMARCA5, while conversely, circSMARCA5 overexpression decreased SMARCA5 levels (Fig. 2a-c and Figure S3). Consistently, the protein of SMARCA5 was high expressed in breast tumor samples as compared with the corresponding controls (Figure S4). Moreover, the ratio of circ-to-linear of circSMARCA5 was significantly lower in breast and renal tumor tissue than the corresponding adjacent tissue specimens (Fig. 2d-e and Figure S1C). Besides, a significant negative correlation was also found between circSMARCA5 and SMARCA5 expression in various cell lines and primary cancer tissues (Fig. 2d-f and Figure S5), which corroborates our observation that circSMARCA5 decreased the expression of SMARCA5 in cancer cells.
cirSMARCA5 terminates the transcription of SMARCA5 at exon 15

We further investigated the mechanism of circSMARCA5 in regulating the expression of SMARCA5. Interestingly, we found that the overexpression of circSMARCA5 indeed decreased the expression of SMARCA5 exons 15-24 but had minimal effects on the expression of exons 1-14 (Fig. 3a). Next, we designed a primer location in exon 13 for the amplification of 3' cDNA ends by rapid amplification of cDNA ends (RACE) PCR (Fig. 3b, left). As shown in Fig. 3b, SMARCA5 can give rise to multiple isoforms. Importantly, we found a decrease in a band of $\sim 5000 \mathrm{bp}$ upon circSMARCA5 overexpression, while an $\sim 250 \mathrm{bp}$ band displayed the opposite phenomenon (Fig. 3b, right). Sanger sequencing showed that the $\sim 5000 \mathrm{bp}$ band and the $\sim 250 \mathrm{bp}$ band are derived from full-length and truncated mRNA (exons 1 to 14), respectively, of the SMAR CA5 gene (Fig. 3c). Consistent with the RACE results, Northern blot assay further demonstrated that ectopic circSMARCA5 expression decreased SMARCA5 levels and promoted truncated mRNA levels (Fig. 3d). The observations gathered thus far have led us to hypothesize that circSMARCA5 prevents transcription from exon 15 of SMARCA5. Indeed, ChIP analysis indicated that the binding of pol II to exons 1-14 of SMARCA5 was higher than that to exons 15-24 (Fig. 3e, left), and the ectopic expression of circSMARCA5 decreased the binding of Pol II to exons 15-24 of SMARCA5 (Fig. 3e, right). To further address whether circSMARCA5 could terminate the transcriptional elongation of SMARCA5, we cloned a series of exons of SMARCA5 in a luciferase plasmid reporter (Fig. 4a, upper). The transient transfection of these luciferase reporters containing the 15-16 exon sequence revealed that luciferase activity was significantly decreased when circSMARCA5 was overexpressed (Fig. 4a, lower). 


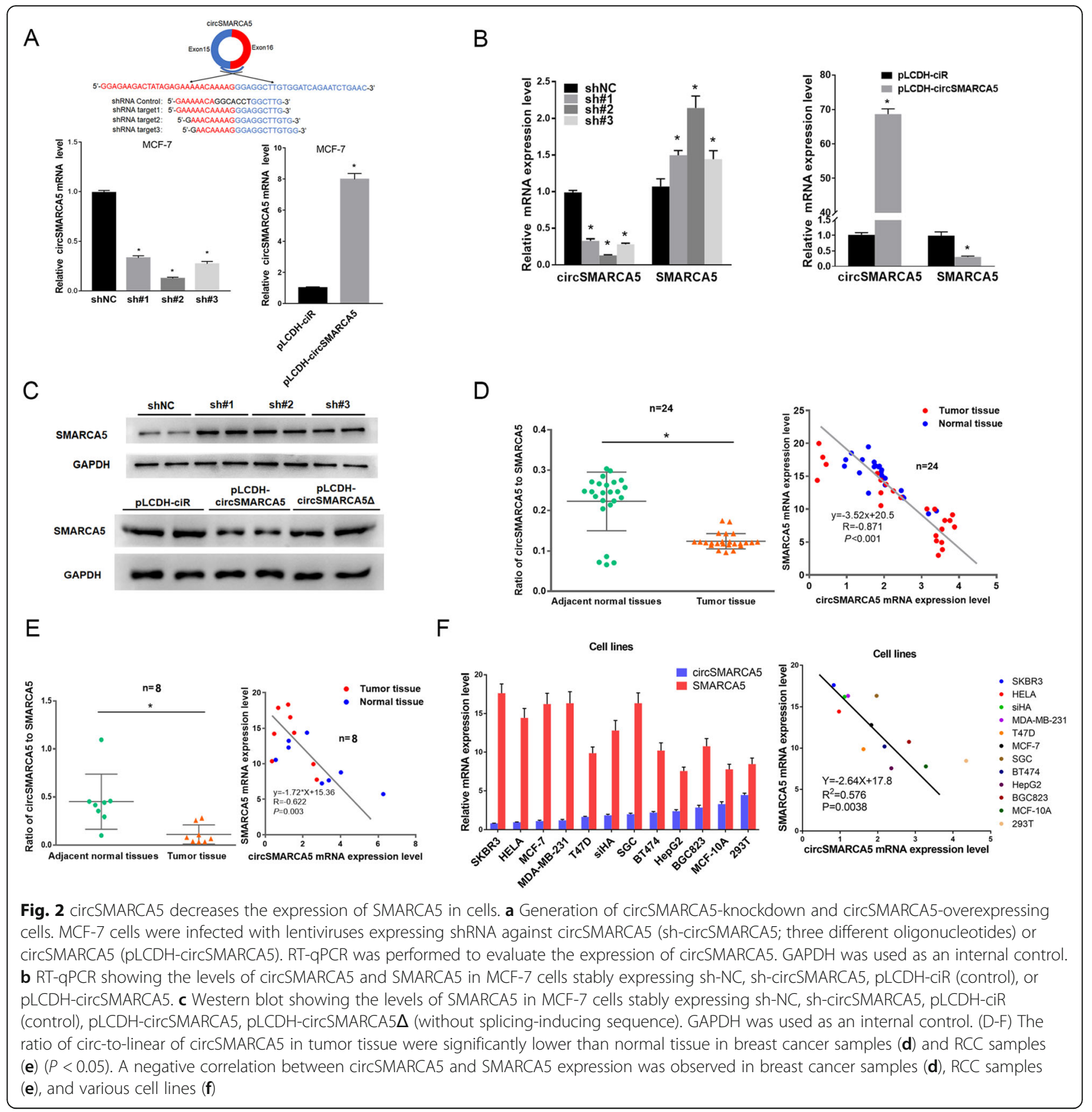

To further confirm the effect of circSMARCA5 on the transcriptional elongation of SMARCA5, we inserted exons of SMARCA5 between DsRED and EGFP as indicated (Fig. 4b, upper). The EGFP level was significantly decreased by circSMARCA 5 when exons $15-16$ were present (Fig. 4b, lower). We further investigated the role of circSMARCA5 in the regulation of SMAR CA5 at the protein level. As expected, circSMARCA5 overexpression downregulated the protein levels of SMARCA5 and upregulated truncated SMARCA5 ( $\triangle$ SMARCA5) protein levels (Fig. 4c and Figure S6), which was confirmed by mass spectrometry (Fig. 4d). Moreover, we found that $\triangle$ SMARCA5 is more susceptible to proteolysis by the proteasome than SMAR CA5 (Fig. 4e). Together, these results show the role of circSMARCA 5 in the termination of transcriptional elongation at exon 15 of SMARCA5.

\section{circSMARCA5 can form R-loops with its parent gene}

To further dissect the mechanism of SMARCA5 transcriptional termination mediated by circSMARCA5, we investigated whether circSMARCA5 can bind genomic 


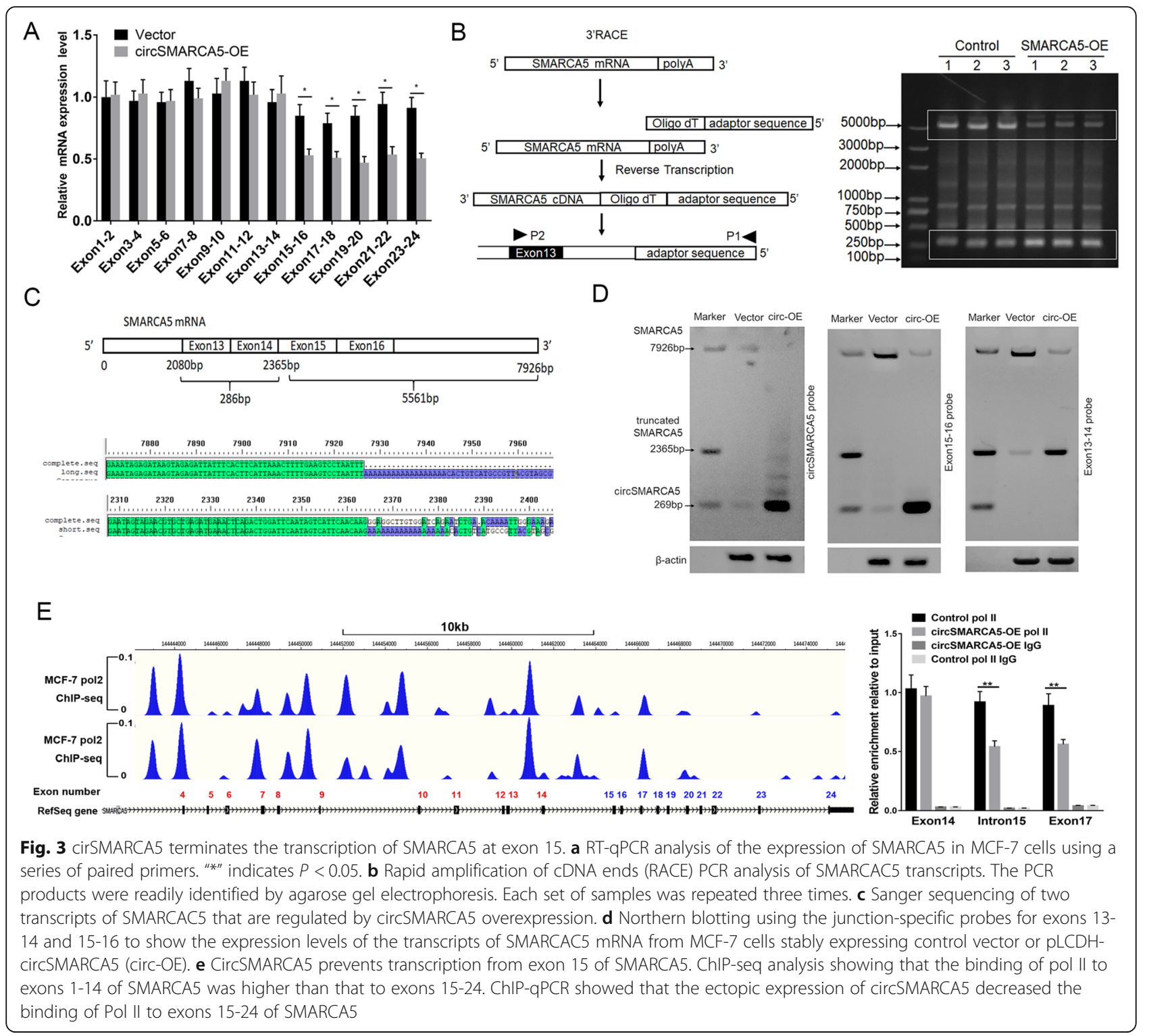

SMARCA5 DNA to form an R-loop. Dot-blotting with R-loop-specific S9.6 antibody supported our hypothesis that circSMARCA5 can bind exons 15-16 of SMARCA5 genomic DNA (Fig. 5a). Additionally, we performed DNA-RNA immunoprecipitation (DRIP) qPCR and confirmed the interaction between circSMARCA5 and exons 15-16; pretreatment with RNase $\mathrm{H}$ ablated this interaction, confirming that the interaction is R-loopspecific (Fig. $5 \mathrm{~b}$ and Figure S7). The interaction of cirCSMARCA5 with the DNA of SMARCA5 was directly verified by fluorescence in situ hybridization (Fig. 5c). Consistent with previous findings [17], dot-blotting of the genome without RNA digest revealed that the binding of circRNA to genomic DNA may be widely present in cancer cells (Fig. 5d). We next determined the specific sequence of exons 15-16 required for R-loop formation.
A series of fragments from exons 15-16 were hybridized with circSMARCA5 for the dot-blotting assay. As shown in Fig. 5e, the $67 \mathrm{bp}$ fragment of the $5^{\prime}$ end of exon 15 plays important role in interacting with circSMARCA5. Moreover, the secondary structure of circSMARCA5 was determined by the software MFOLD [29], which revealed the sequence 5 '-AACAAAAUUGGGAAAGAU GAAAUGCUUCAAAU- 3 ' from the $5^{\prime}$ end of exon 15 located in the loop region of circSMARCA5 (Fig. 6a). We thus hypothesized that this sequence might play a key role in mediating the circSMARCA5-DNA interaction. To this end, we synthesized the wild-type and mutant phosphorylated DNA fragments, ANT and ANT-mut, respectively, corresponding to this sequence (Fig. 6b). Dot-blotting demonstrated that wild-type oligonucleotides (ANT) can bind to circSMARCA5, but 


\section{A}

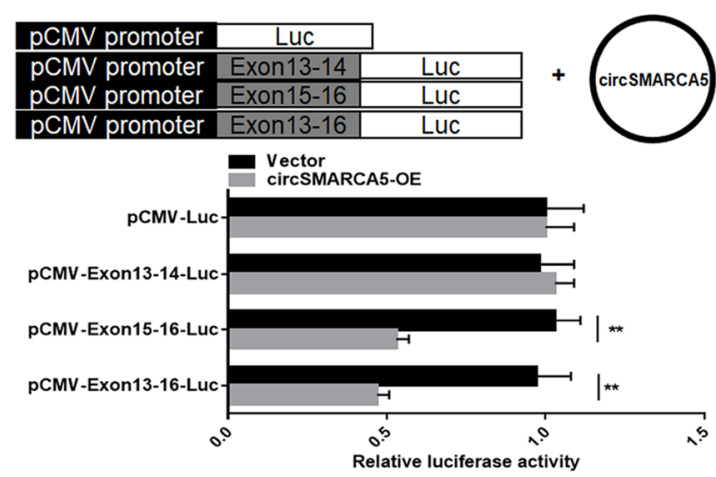

B

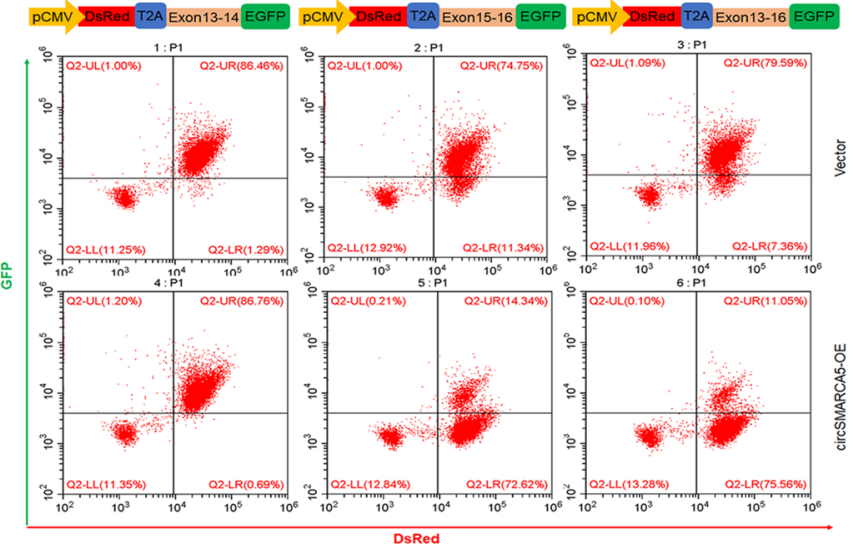

C
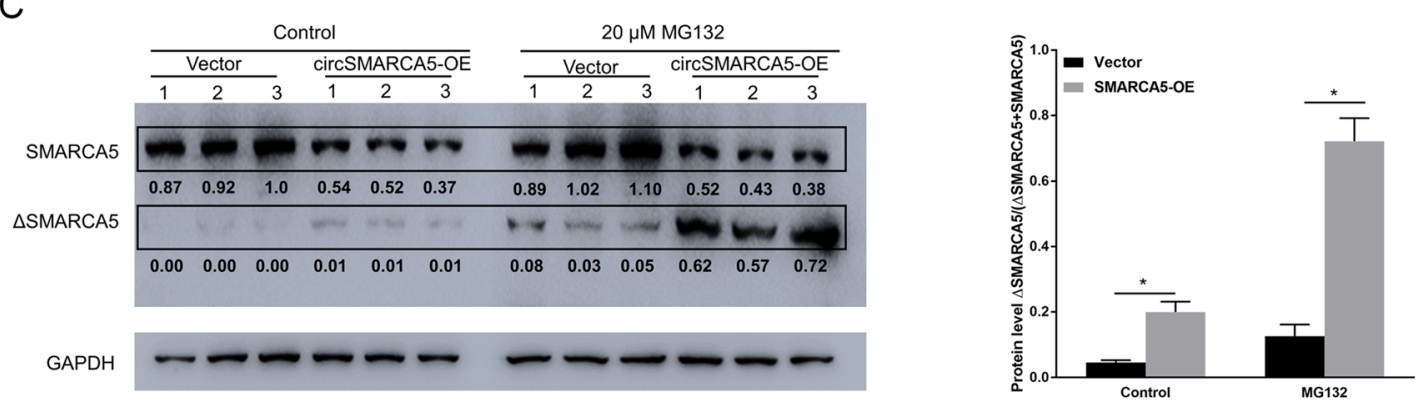

D

SMARCA5 protein sequence: $\mathrm{N}^{\mathrm{r}}$ -

MSSAAEPPPPPPPESAPSKPAASIASGGSNSSNKGGPEGVAAQAVASAASAGPADAEMEEIFDDASPGKQKEIQEPDPTYEEKMQTDRANRFEYLLKQTELFAHFIQPAAQKTPTSPLKMKPGRPRIKKDEKQNLLSVGDYF EII Q Q E P D D P T T Y IEE IK

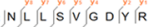

HRRTEQEEDEELLTESSKATNVCTRFEDSPSYVKWGKLRDYQVRGLNWLISLYENGINGILADEMGLGKTLQTISLLGYMKHYRNIPGPHMVLVPKSTLHNWMSEFKRWVPTLRSVCLIGDKEQRAAFVRDVLLPGEWDVCV

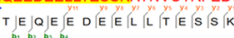

TSYEMLIKEKSVFKKFNWRYLVIDEAHRIKNEKSKLSEIVREFKTTNRLLLTGTPLQNNLHELWSLLNFLLPDVFNSADDFDSWFDTNNCLGDQKLVERLHMVLRPFLLRRIKADVEKSLPPKKEVKIYVGLSKMQREWYTRILM YLIVIT DOEFAHR

KDIDILNSAGKMDKMRLLNILMQLRKCCNHPYLFDGAEPGPPYTTDMHLVTNSGKMVVLDKLLPKLKEQGSRVLIFSQMTRVLDILEDYCMWRNYEYCRLDGQTPHDERQDSINAYNEPNSTKFVFMLSTRAGGLGINLATAD D I D D L L N S S A TGKK

WILYDSDWNPQVDLQAMDRAHRIGQTKTVRVFRFITDNTVEERIVERAEMKLRLDSIVIQQGRIVDQNLNKIGKDEMLQMIRHGATHVFASKESEITDEDIDGILERGAKKTAEMNEKLSKMGESSLRNFTMDTESSVYNFEG

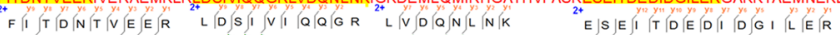

EDYREKOKIAFTEWIEPPKRERKANYAVDAYFREALRVSEPKAPKAPRPPKQPNVQDFQFFPPRLFELLEKEILFYRKTIGYKVPRNPELPNAAQAQKEEEQLIDEAESLNDDEELEEKEKLLTQGFTNWNKRDFNQFIKANEKW GRDDIENIAREVEGKTPEEVIEYSAVFWERCNELQDIEKIMAQIERGEARIQRRISIKKALDTKIGRYKAPFHQLRISYGTNKGKNYTEEEDRFLICMLHKLGFDKENVYDELRQCIRNSPQFRFDWFLKSRTAMELQRRCNTLIT LIERENMELEEKEKAEKKKRGPKPSTQKRKMDGAPDGRGRKKKLKL -C

$\mathrm{E}$

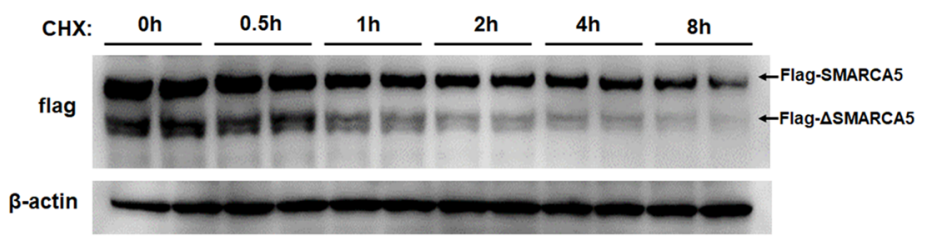

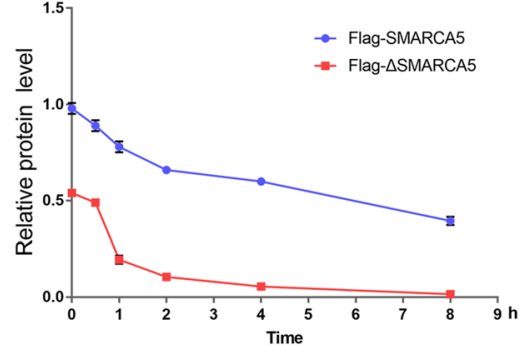

Fig. 4 (See legend on next page.) 
(See figure on previous page.)

Fig. 4 cirSMARCA5 blocks the transcription of SMARCA5 and promotes the generation of a truncated SMARCA5 protein ( $\triangle S M A R C A 5)$. a Schematics of luciferase reporter constructs containing the SMARCA5 exon sequence as indicated (upper). The SMARCA5 exon 15-16 sequence plays an important negative role in mediating the effect of circSMARCA5 overexpression on luciferase activity (lower). b Schematics of fluorescence reporter constructs containing the SMARCA5 exon sequence as indicated (upper). MCF-7 cells were transiently transfected with these fluorescence reporters along with or without circSMARCA5 Co-overexpression. After transfection for 48 hours, the reporter transcription activities were measured by flow cytometry assay. c circSMARCA5 overexpression downregulated the protein levels of SMARCA5 while upregulating truncated SMARCA5 ( $\triangle$ SMARCA5) protein levels. MCF-7 cells stably overexpressing circSMARCA5 or control cells were treated with DMSO or MG132. Western blot analysis was performed using an antibody targeting the N-terminus of SMARCA5 to evaluate the expression of SMARCA5 and $\triangle$ SMARCA5. GAPDH was used as an internal control. $\mathbf{d}$ The $\triangle$ SMARCA5 protein was identified by mass spectrometry, and detected SMARCA5 peptides were showed in the map. The red-labeled portion is the amino acid sequence of the translated defective transcript. e MCF-7 cells expressing Flag-SMARCA5 and Flag- $\triangle$ SMARCA5 were treated with cycloheximide (CHX, $50 \mu \mathrm{g} / \mathrm{ml})$. The cell lysates were subsequently harvested at sequential time points $(0,0.5,1,2,4$ or $8 \mathrm{~h})$ after treatment, and then the cell lysates were immunoblotted with anti-Flag or antiActin antibody

mutant oligonucleotides (ANT-mut) cannot bind to circSMARCA5 (Fig. 6c). As expected, DRIP-qPCR showed that ANT inhibited circSMARCA5 binding to the DNA at exons 15-16, whereas ANT-mut had no effect on this interaction (Fig. 6d). Furthermore, the transfection of ANT prevented the decrease in SMARCA5 protein levels in MCF-7 cells stably expressing circSMARCA5, whereas ANT-mut had no effect on SMARCA5 protein levels (Fig. 6e). Importantly, the mutation of the key sequence in circSMARCA5 impaired the interaction with its parent gene, which was confirmed by dotblotting and DRIP-qPCR assays (Fig. 6f-h and Figure S8). Unlike circSMARCA5, circSMARCA5-mut had little effect on SMARCA5 protein levels (Fig. 6i). These results suggested that circSMARCA5 formed R-loops with its parent gene to inhibit the expression of SMAR CA5 in cancer cells.

\section{circSMARCA5 inhibits DNA damage repair function}

To explore the roles of circSMARCA5 in cancer progression, we overexpressed and depleted circSMARCA5 in MCF-7 cells by lentiviral vectors and then examined the effect of circSMARCA5 on cell proliferation, migration, and apoptosis. However, the results showed that both overexpressed and depleted circSMARCA5 had no effect on these three activities (Figure S9). Previous studies have indicated that SMARCA5 plays an important role in regulating the DNA repair process and maintaining the stability of the genome [22, 30-32]. Consistent with previous reports, SMARCA5 overexpression improved DNA repair capacity and reduced the expression of Chk1 and Chk2 after DNA damage repair (Figure S10A). Given that circSMARCA5 can promote the production of the truncated $\triangle$ SMARCA5 protein, we tested whether the truncated protein is also functional. The overexpression of Flag- $\triangle$ SMARCA5 had a minimal effect on the expression of Chk1 and Chk2 after DNA damage repair (Figure S10B), suggesting that $\triangle$ SMARCA5 is a nonfunctional protein product. We next assessed whether circSMARCA5 can affect the function of DNA damage repair capacity. CCK8 and clone formation assays revealed that circSMARCA5 overexpression increased sensitivity to cisplatin or bleomycin in MCF-7 cells (Fig. 7a, b). Next, MCF-7 cells were treated with the indicated concentration of cisplatin or bleomycin for $24 \mathrm{~h}$ and then the DNA damage was evaluated by single cell gel electrophoresis (SCGE) at 48 and $72 \mathrm{~h}$. MCF-7 cells expressing circSMARCA5 showed significantly lower repair capacity than did control cells (Fig. 7c). In parallel, DNA damage was examined after $72 \mathrm{~h}$ of treatment with cisplatin or bleomycin by using an anti- $\gamma \mathrm{H} 2 \mathrm{AX}$ antibody. Consistent with the SCGE results, the $\gamma \mathrm{H} 2 \mathrm{AX}$ signal in MCF-7 cells expressing circSMARCA5 was significantly higher than that in MCF-7 cells, as evidenced by immunostaining (Fig. 7d). Consistently, cisplatin significantly enhanced the levels of DNA damage response proteins Chk1 and Chk2 in MCF-7 cells expressing circSMARCA5 (Fig. 7e), whereas several key cell-cycle genes were reduced specifically upon circSMARCA5 overexpression (Fig. 7f). To test whether circSMARCA5 R-loop formation is necessary for its DNA repair function, we transfected ANT or ANT-mut into circSMARCA5-expressing cells. The SCGE assay and $\gamma \mathrm{H} 2 \mathrm{AX}$ measurement showed that ANT significantly enhanced the DNA repair capacity, while ANT-mut had no effect on this activity (Fig. $7 \mathrm{~g}$ and Figure S11). Furthermore, ANT significantly decreased the degree of colocalization between cirCSMARCA5 and its cognate DNA locus (Figure S12). In addition, unlike circSMARCA5, the overexpression of circSMARCA5-mut had little effect on the DNA repair rate (Fig. 7h and Figure S13A, B). Next, we determined whether SMARCA5 could mediate the effects of circSMARCA5 in preventing DNA damage repair. As shown in Fig. 7i, the $\gamma \mathrm{H} 2 \mathrm{AX}$ signal was much lower in circSMARCA5-expressing cells complemented with SMARCA5 than that in cells expressing circSMARCA5 alone. As expected, $\triangle$ SMARCA5 could not rescue the inhibition of DNA damage repair function induced by 


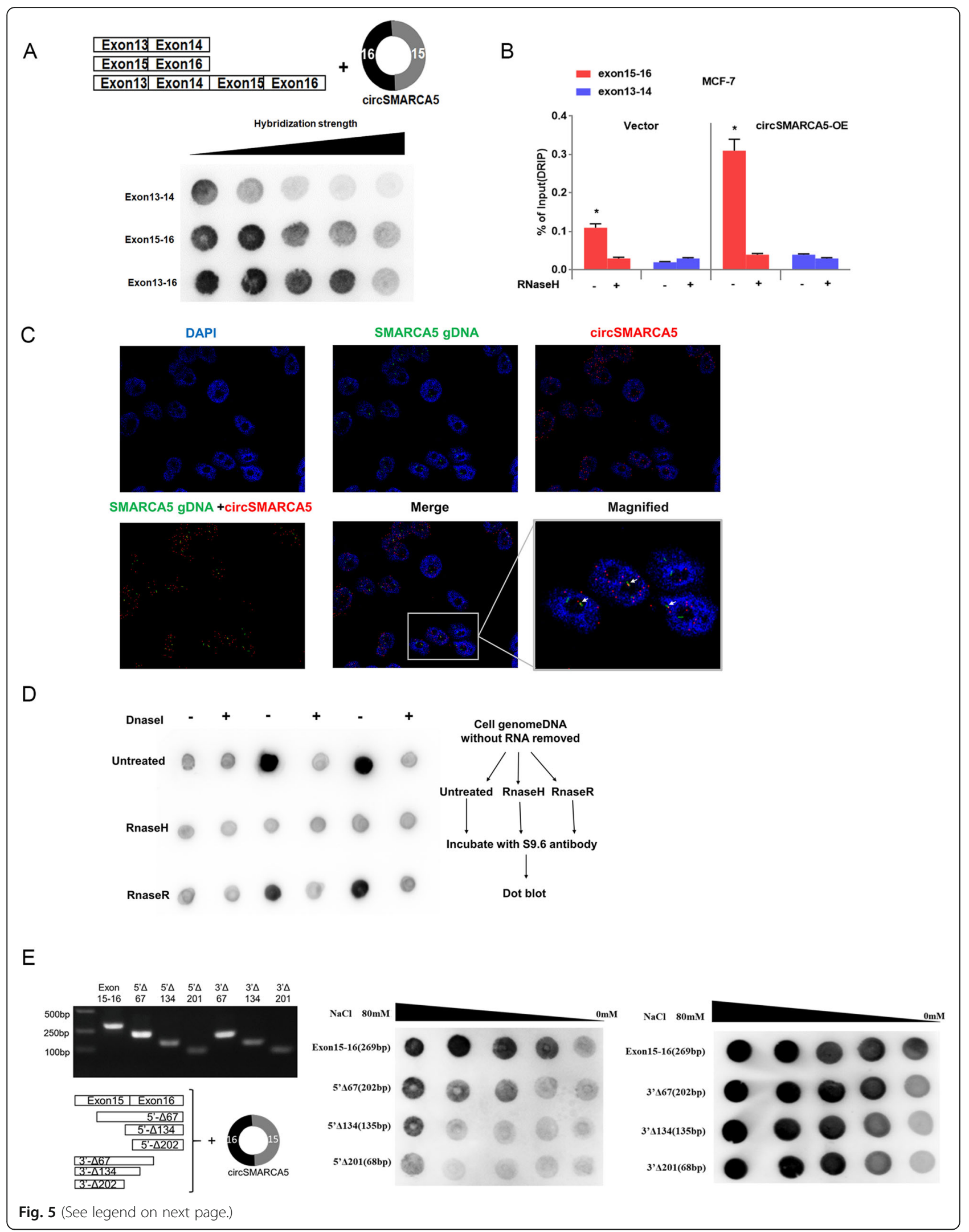


(See figure on previous page.)

Fig. 5 circSMARCA5 interacts with its site of transcription. a circSMARCA5 interacts with the 15-16 exon sequence of the SMARCA5 locus. A series of exon DNA fragments were hybridized with circSMARCA in vitro. The DNA-RNA hybridization strength was quantified by dot-blot with R-loopspecific 59.6 antibody. Hybridization stringency was altered by decreasing ionic strength (80-0.1 mM NaCl). b DRIP-qPCR analysis of the 15-16 exon sequence of SMARCA5 to detect the association of circSMARCA5 in MCF-7 cells. RNase H-treated and/or DRIP-qPCR analysis of the 15-16 exon sequence as a control. c CircSMARCA5 partially localized at its site of transcription. Double FISH of circSMARCA5 (red) and its parent DNA region (green). The nucleus was stained by DAPI. $\mathbf{d}$ Dot-blot of R-loops in MCF-7 cell genomic DNA preparations treated with DNase I, RNase H, or RNase R. The DNA-RNA hybrids in genome DNA were analyzed by S9.6 antibody. e Mapping of the R-loop formation region of circSMARCA5. A series of exon 15-16 deletion mutants were hybridized with circSMARCA5 for the dot-blotting assay. The DNA-RNA hybridization intensity was analyzed by dot-blot with an $\mathbf{5 9 . 6}$ antibody targeting the DNA-RNA hybrid strand. Hybridization stringency was altered by decreasing ionic strength (80-0.1 mM NaCl)

CircSMARCA5 (Figure S13C, D). Moreover, the overexpression of SMARCA5 could significantly rescue the growth defects of cells expressing circSMARCA5, as demonstrated by a colony formation assay (Fig $7 \mathrm{j}$ ). Together, these results demonstrated the roles of circSMARCA5 in regulating the DNA repair process in MCF-7 cells.

\section{circSMARCA5 overexpression enhances the cisplatin response in breast cancer}

To further evaluate the therapeutic potential of circSMARCA5 in breast cancer in vivo, we established circSMARCA5 overexpression clones in MCF-7 cells. As shown in Fig. 8a, the overexpression of circSMARCA5 efficiently enhanced the sensitivity of MCF-7 xenografts to concurrent cisplatin treatment (Fig. 8a, b). The overexpression of circSMARCA5 was confirmed by in situ hybridization and qPCR analysis (Fig. 8c), along with decreased SMARCA5 protein levels and increased $\gamma \mathrm{H} 2 \mathrm{AX}$ levels (Fig. 8d). In addition, qPCR analysis demonstrated that circSMARCA5 can be detected in the blood, suggesting that circSMARCA5 is a secretory molecule. Collectively, these data demonstrate that circSMARCA5 could serve as a potential therapeutic target to restore sensitivity to cisplatin therapy in breast cancer.

\section{Discussion}

Previous studies have indicated that circRNAs have multiple functions in cancer development and progression [33-35]. In this study, we identified multiple expressed circRNAs in breast cancer samples and observed average higher abundance of circRNAs over their host genes in peripheral blood than tissues, which might contribute to the exploration of diagnostic biomarker for breast cancer. We then identified that circSMARCA5 is significantly decreased in breast cancer tissues using RNA-seq. More importantly, we define a critical role for circSMARCA5 in the regulation of DNA damage repair capacity and the drug sensitivity of breast cancer cells in vitro and in vivo through the negative regulation of its parent gene SMARCA5. These findings are of high clinical relevance because chemotherapy with cisplatin and bleomycin remains the standard of care in breast cancer [36-38]. Hence, the restoration of circSMARCA5 levels provides an approach to overcome treatment resistance in breast cancer patients.

SMARCA5, also known as SNF2H, is a member of the SWI/SNF chromatin-remodeling complex. During DNA damage repair processes, SMARCA5 is recruited to DNA damage sites where it induces the ubiquitination and phosphorylation of histone $\mathrm{H} 2 \mathrm{~A}$, which facilitates chromatin remodeling and DNA damage repair $[18,19]$. In this study, we show that circSMARCA5 expression resulted in the downregulation of SMARCA5, and the effect of circSMARCA5 overexpression on DNA repair capacity was reversed by concomitant SMARCA5 overexpression, suggesting that the effect of circSMARCA5 on DNA repair capacity is mediated through SMARCA5. circRNAs exert functions in various ways, such as forming an R-loop with DNA to regulate splicing and transcriptional pausing [17]. For example, circSEPALLATA3 regulates the splicing of its parent mRNA through $\mathrm{R}$ loop formation [17]. In addition, circRNAs are a novel class of ceRNAs that sponge miRNAs, thus positively regulating gene expression [13, 14]. Additionally, circRNAs, such as exon-intron circRNAs, regulate gene expression through specific RNA-RNA interactions with U1 snRNA [39]. Furthermore, circRNAs also exert functions by binding to proteins and regulating their activities [40]. We identified one mechanism by which circSMARCA5 regulates the drug sensitivity of breast cancer cells to cisplatin and bleomycin through the downregulation of SMARC5. circSMARCA5 is recruited to its parent gene locus, leading to R-loop formation, transcription termination, nonfunctional truncated $\triangle$ SMARCA5 protein upregulation, and decreased SMAR CA5 expression. This regulatory mechanism has also been verified in cervical cancer (Hela cells) (Figure S14). However, our evidence demonstrates that circSMARCA5 has no significant effect on the proliferation, migration and apoptosis of breast cancer cells, suggesting that this molecule functions in a cell-type and context-dependent manner. Notably, we provide evidence that 


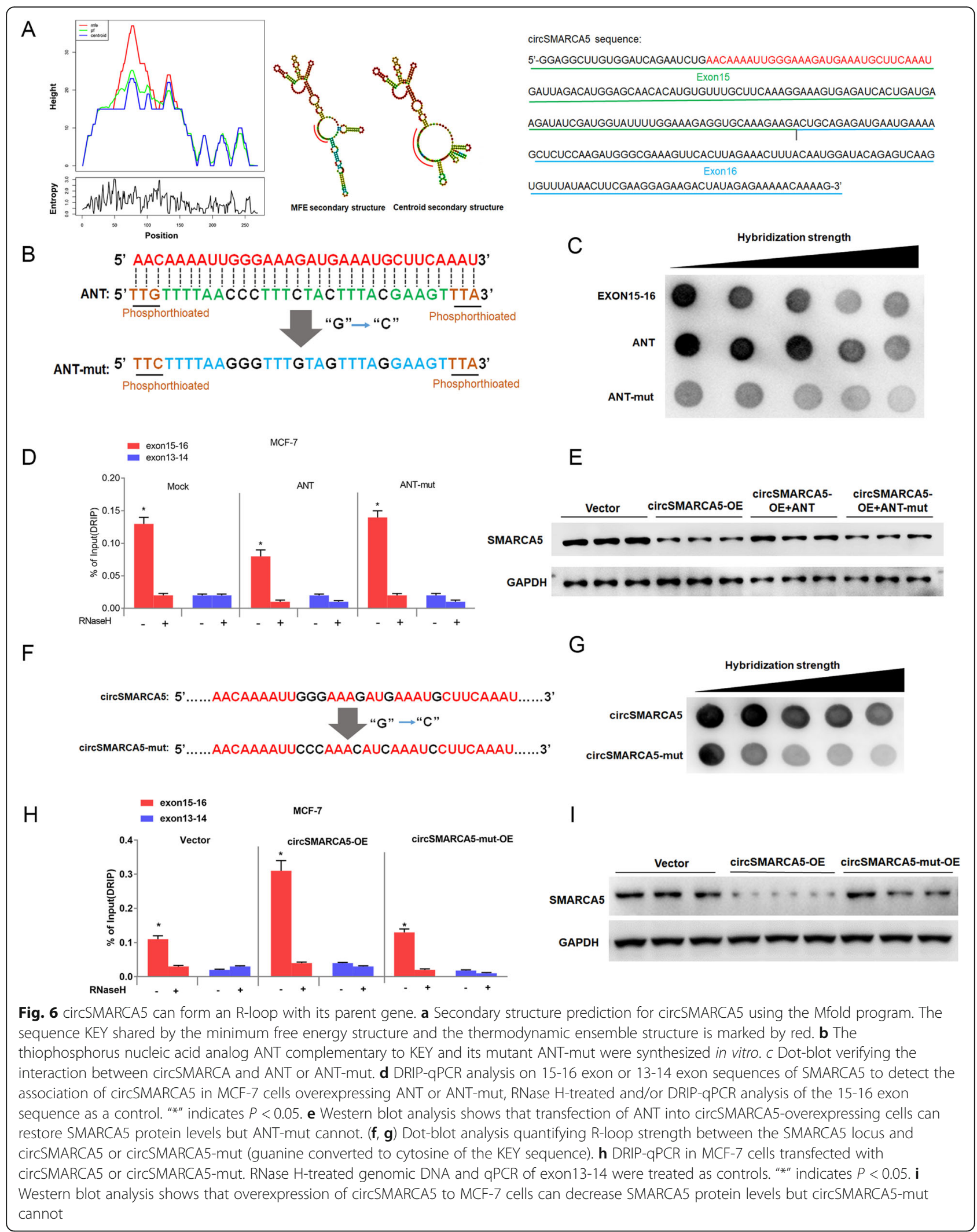




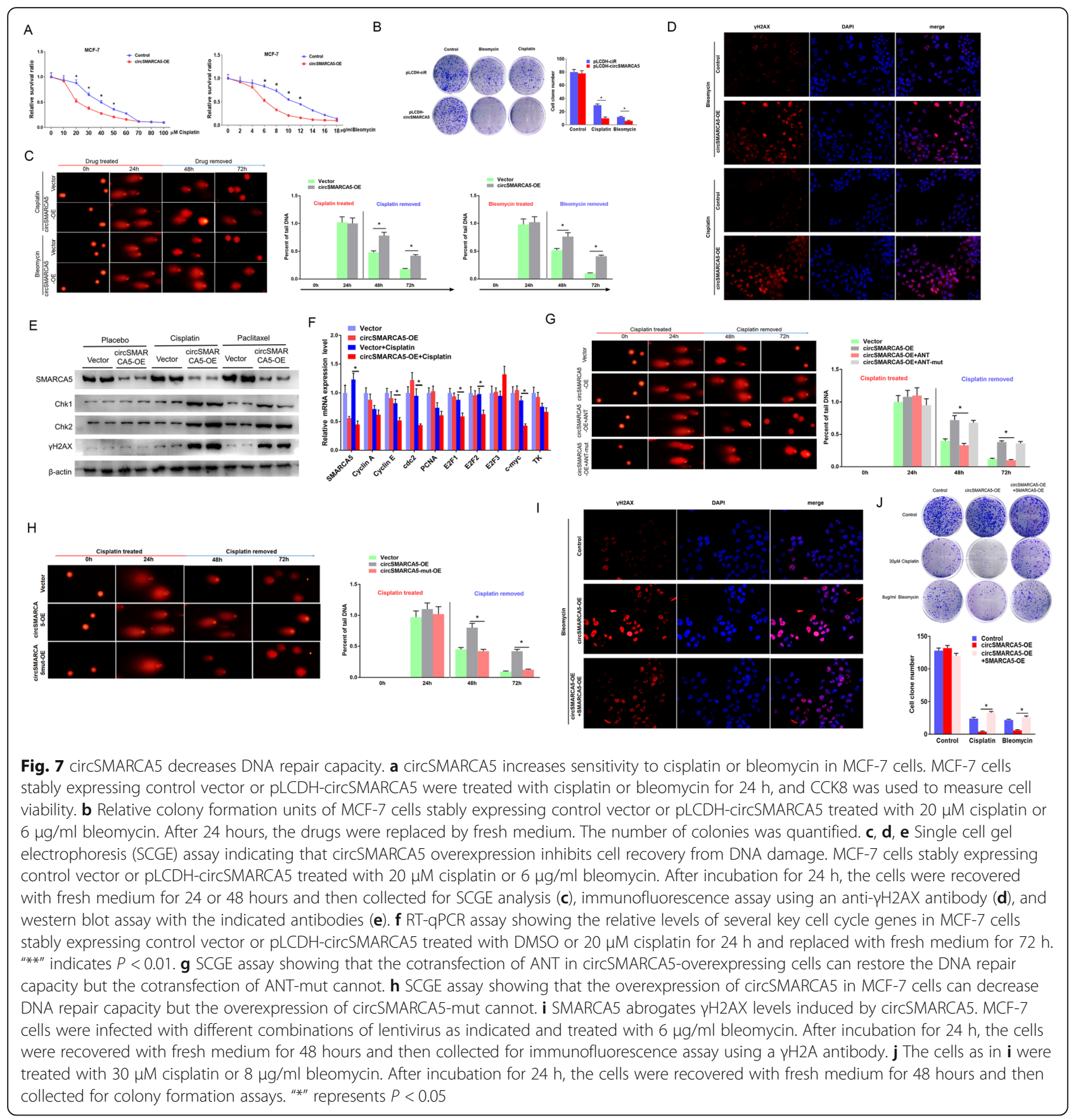

circSMARCA5 plays a key role in the DNA repair pathway and drug resistance, making it a promising therapeutic target for breast cancers.

CircSMARC5 has been shown to be downregulated in hepatocellular carcinoma tissues, and its downregulation is associated with aggressive characteristics and unfavorable outcomes [25]. We also confirmed a low abundance of circSMARCA5 in breast cancer and renal cancer tissues compared with matched adjacent normal tissues.

Due to the features of circRNA, including stability, tissue specificity and abundance in bodily fluids,
circRNAs may serve as potential biomarkers for various diseases [3, 41, 42]. Altogether, the findings in this study confirmed that the overexpression of circSMARCA 5 was sufficient to improve the chemosensitivity of breast cancer cells in vitro and in vivo, indicating the important regulation mechanism of this circRNA in breast cancer. And our analysis revealed the negative correlation is also existed in other cancers, suggesting a general regulation of circSMARCA 5 on host gene in transformed cells. Furthermore, we found that the ratio of circ-to-linear (expression of circRNA / linear host genes) of 
A
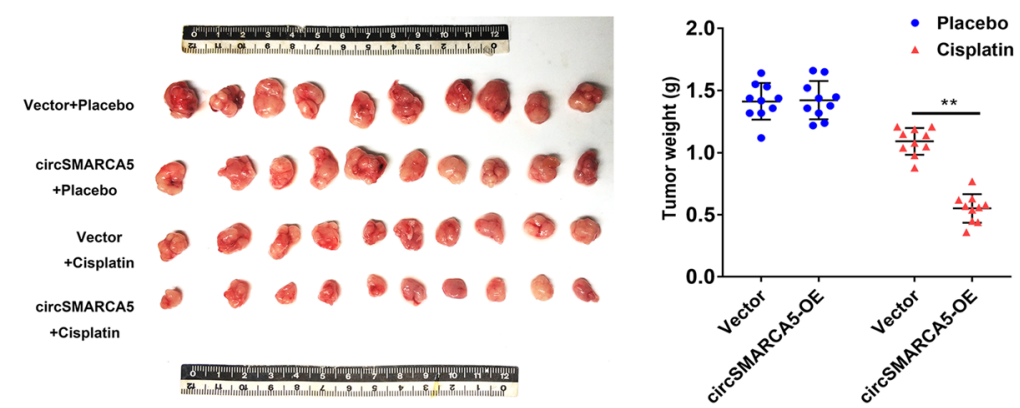

B

C
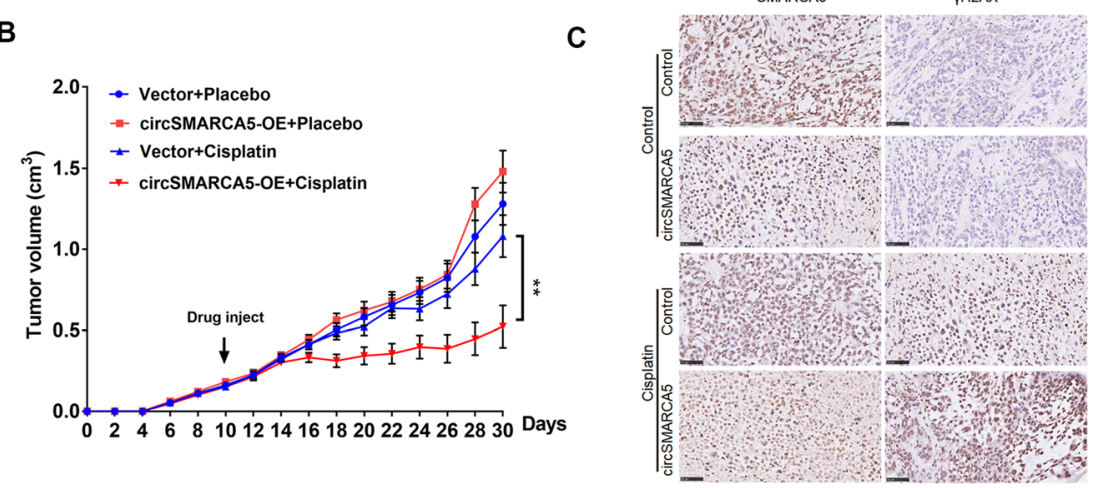

D

E
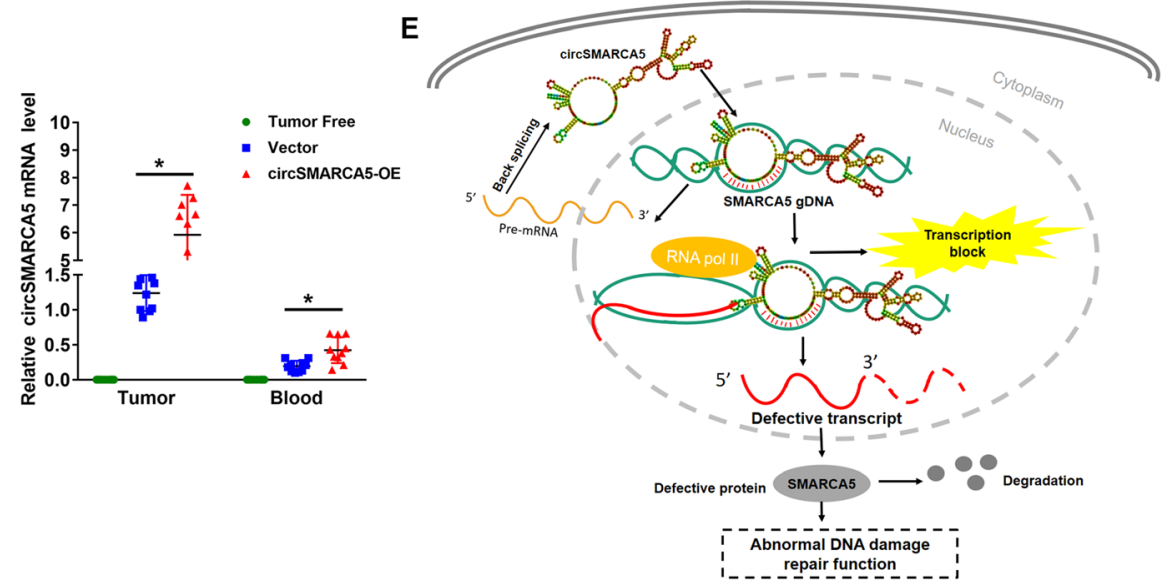

Fig. 8 Figure 8. circSMARCA5 overexpression enhances the cisplatin response in breast cancer. a MCF-7 cells expressing control vector or pLCDHcircSMARCA5 were transplanted into mice. After the average tumor volume reached $0.3 \mathrm{~cm} 3$, the mice were injected with cisplatin or placebo. (Left) Representative images of the isolated tumors from injected mice. (Right) Tumor weight was calculated according to the formula provided in the Materials and Methods section. b After injection with cisplatin or placebo, the mean tumor volume was measured by calipers on the indicated days. c Representative immunofluorescence staining images (scale bars, $100 \mu \mathrm{m}$ ) and SMARCA5 and $\mathrm{HH} 2 \mathrm{AX}$ expression in xenograft tumors derived from MCF-7 cells as in A. $\mathbf{d}$ RT-qPCR assay showing the relative levels of circSMARCA5 in the tumor tissues and blood of xenograft mice. The blood of tumor-free mice was treated as a control. "**" represents $P<0.05$. e Proposed model for circSMARCA5

downregulation of its parent gene expression

circSMARCA5 in the blood sample of breast cancer patients were significantly lower than health volunteers (Fig. 1c and S1B), which indicating that circSMARCA5 may serve as an indicator for the evaluation of breast cancer liquid biopsy. Further clinical research is needed to clarify whether circSMARCA5 is a useful biomarker in breast cancer.

\section{Conclusion}

We observed average higher expression of circRNAs than their host genes in blood samples comparing to breast cancer and adjacent normal tissues. And for the first time, we validated the interaction between circRNA and its host gene DNA in cancer, through a regulation model of circRNA and the transcription of partial exons 
of host gene. Furthermore, we found circRNA could affect the sensitivity of tumor to chemotherapy drug and our result indicated this circRNA is involved in DNA damage repair in human cancer. Our results revealed a new regulatory mechanism for circRNA on its host gene and provided evidence that circSMARCA5 may serve as a therapeutic target for drug-resistant breast cancer patients.

\section{Materials and methods}

\section{Cell culture and cell transfection}

All cell lines, including $293 \mathrm{~T}, \mathrm{MCF}-10 \mathrm{~A}, \mathrm{MCF}-7$, SKBR3, BT474, T47D and MDA-MB-231, were purchased from the China Center for Type Culture Collection (CCTCC, Chinese Academy of Sciences, Shanghai, China). The cells were cultured according to the protocols from the American Type Culture Collection (ATCC; https:// www.atcc.org/). Plasmid and phosphorylated deoxyribonucleic acid single strand transfection were carried out by Zlipo2000 (Zomanbio, Beijing, China) or liposomal transfection reagent (Yeasen, Shanghai, China).

\section{Sample collection}

Breast cancer tissues, adjacent tissues and blood samples were collected from breast cancer patients treated at Zhongnan Hospital of Wuhan University (Wuhan, China) after written informed consent was obtained. One milliliter of EDTA blood from different breast cancer patients was collected before surgery. The blood samples were stored in Trizol at $-80^{\circ} \mathrm{C}$ until use. In the RNA-seq study, 6 women (mean age: 52 years, range: 45-60 years) diagnosed with luminal-B subtype breast cancer were included. The amplification and overexpression of HER2 were found in 3 patients, while the other patients were negative in HER2 status. In addition, cancer and adjacent normal tissues and blood samples from 24 breast cancer patients with clinical pathologic information, and blood samples from 24 health volunteers were collected for the qPCR validation.

\section{Whole transcriptome sequencing}

Total RNA was extracted from the tumor, adjacent normal tissue and blood samples of six breast cancer patients, followed by rRNA depletion from total RNA. Then, the RNA was reverse transcribed to cDNA and constructed into a strand-specific library. Illumina HiSeq XTen was performed for sequencing. All raw data can be accessed in the NCBI GEO database (https://www. ncbi.nlm.nih.gov/geo/query/acc.cgi?acc=GSE133998, accession code: wxmjuumulvsbbuf).

\section{Identification of circRNA expression}

To identify the expression of circRNAs from RNA-Seq, one extensively used algorithm Circexplorer3 [43] were utilized for detecting the back-splice junction sites of circRNAs. And the fragments per billion mapped bases (FPB) of circRNA and its linear host gene were extracted to calculate the CIRCscore (FPBcirc/FPBlinear), which could indicate the expression ratio of circRNA to their host genes. Genome assembly GRCh 37 and GENCODE (version 19) gene annotation were used. Total 8312 circRNAs were detected with expression across all six patients.

\section{Northern blot analysis}

Northern blot (NB) analysis was performed according to the manufacturer's instructions (DIG Northern Starter Kit, Roche). Digoxigenin (DIG)-labeled riboprobes were transcribed by DIG RNA labeling Mix (Roche, USA) and the HiScribe T7 In Vitro Transcription Kit (NEB, USA), and then purified by phenol-chloroform extraction and ethanol precipitation. Ten micrograms of total RNA or $1 \mathrm{mg}$ of in vitro synthesized linear or circRNA was electrophoresed on a denaturing urea polyacrylamide gel, transferred to a Hybond-N+ membrane (GE, USA) by a positive capillary transfer system and UV-crosslinked as a standard protocol. The membrane was then hybridized with specific DIG-labeled riboRNA probes. NB probes are listed in Table S2.

DNA and RNA fluorescence in situ hybridization (FISH)

The cells were fixed with $4 \%$ paraformaldehyde. After permeabilization with proteinase $\mathrm{K}$ pretreatment, the DNA was denatured with $2 \mathrm{X}$ SSC solution containing $70 \%$ formamide at $72{ }^{\circ} \mathrm{C}$ and then incubated for $12 \mathrm{~h}$ at $37^{\circ} \mathrm{C}$ in hybrid solution [ $50 \%$ formamide, $2 \mathrm{X}$ SSC, 0.25 $\mathrm{mg} / \mathrm{mL}$ Escherichia coli transfer RNA, $0.1 \mathrm{mg} / \mathrm{mL}$ salmon sperm DNA (Sigma, USA), $2.5 \mathrm{mg} / \mathrm{mL}$ BSA (Sigma, USA), and DIG- or biotin-labeled $100 \mathrm{pM}$ probes transcribed by T7 In Vitro Transcription Kit (NEB, USA)]. After washing with hybrid solution supplemented with RNase A to remove excessive probes, the cells were subjected to indirect immunofluorescence using FITC/ AF594 coupled with anti-DIG antibody or FITC/AF594 coupled with streptavidin. The nuclei were stained with DAPI. The probes are listed in Table S2.

\section{Plasmid constructs}

The sequence for exons 15-16 of SMARCA5 was PCR amplified using primers $F$ (5'-CGGAATTCTG AAATATGCTATCTTACAG GGAGGCTTGTGGAT CAGAAT.

CTG-3') and $\mathrm{R}$ (5'-CGGGATCCTCAAGAAAAA ATATATTCACCTTTTGTTTTTC.

TCTATAGTCTCTCC - 3'), digested by EcoRI and BamHI, and ligated into pLCDH-ciR (Geneseed, China) and pLCDH-ciR-GFPmut (GFP mutated by site-directed mutagenesis) to create two circSMARCA5-overexpressing 
plasmids. The pCMV-DsRed-T2A-MSC-EGFP reporter vector was constructed by cloning the coding region of DsRed and T2A into psi-EGFP-N1(Addgene, USA). Exons 13-14, 15-16 and 13-16 of SMARCA5 were PCR amplified and cloned into the pCMV-DsRed-T2A-MSC-EGFP reporter by in-Fusion cloning. To overexpress SMARCA5 and $\triangle$ SMARCA5 protein, the SMARCA5 complete and defective transcript sequences were cloned into pFLAGCMV2 (Addgene, USA). Gene-specific shRNA target sequences were synthesized and cloned into the pLKO.1 plasmid. The primers for making these constructs are provided in Table S3.

\section{RNA secondary structure prediction}

The circSMARCA5 complete sequence was input into the RNAfold web server (http://rna.tbi.univie.ac.at/cgibin/RNAWebSuite/RNAfold.cgi). The minimum free energy structure and thermodynamic ensemble structure were predicted by this website.

\section{The $3^{\prime}$ rapid amplification of CDNA ends ( $3^{\prime}$ RACE)}

The total RNA was extracted from cells, and the integrity was verified. Oligo dT with a tag sequence was used for reverse transcription. The $3^{\prime}$ end sequences of mRNA were PCR amplified using primers located in SMARCA5 exon 2 and the tag sequence. Fragments with different expression levels were identified by one-generation sequencing. All primers are listed in Table S3.

\section{R-loop dot-blotting}

Dot-blotting was performed using S9.6 monoclonal antibody according to previously published protocols [17, 44]. DNase I, RNase H and RNase R (Epicentre Technologies) treatments were performed essentially as described using a standard hybridization buffer $(10 \mathrm{mM}$ Tris- $\mathrm{HCl}, \mathrm{pH} 7.5,50$ $\mathrm{mM} \mathrm{NaCl}, 0.1 \mathrm{mM}$ EDTA). RNA for exons 13-14, 15-16 and 15-16 mutation templates of SMARCA5 were transcribed with a T7 RNA Polymerase kit (NEB, USA) and circularized by splint ligation with T4 RNA ligase with circRNAs purified from linear RNAs by RNase R digestion. DNA templates, including SMARCA5 exons 13-14, 15-16, 13-16 and their mutation templates, were amplified by PCR. The strength of the R-loops was assessed by increasing the hybridization stringency through a stepwise decrease in ionic strength from 80 to $10 \mathrm{mM} \mathrm{NaCl}$. The dot-blot experiments were repeated three times, with quantification performed with Image Lab software ver 5.2.1 (mean, background normalized values presented).

\section{Drip}

DRIP was carried out as described [45] with some modifications. In brief, $40 \mu \mathrm{g}$ of genomic DNA was extracted from cells by PCI (phenol:chloroform:isoamyl alcohol =
24:23:1), with $20 \mu \mathrm{g} / \mathrm{ml}$ proteinase $\mathrm{K}$ (Sigma, USA) and $20 \mu \mathrm{g} / \mathrm{ml}$ RNase A (Sigma, USA) applied to degrade proteins and RNA in the genome, respectively. The DNA was digested with $6 \mathrm{U}$ EcoRI, EcoRV, XbaI, BamHI, and SspI (NEB, USA) at $37^{\circ} \mathrm{C}$ overnight or treated with 0.4 $\mathrm{U} / \mu \mathrm{l}$ RNase H (Diamond, China) as a negative control for R-loop validation. The purified DNA fragment at $10 \mu \mathrm{g}$ was incubated with $10 \mu \mathrm{g} \mathrm{S9.6}$ antibody (Kerafast, USA) at $4{ }^{\circ} \mathrm{C}$ for $2 \mathrm{~h}$ in $0.2 \mathrm{ml}$ IP buffer (20 mM HEPES$\mathrm{KOH}$ (pH 7.5), $150 \mathrm{mM} \mathrm{NaCl}, 10 \mathrm{mM} \mathrm{MgCl} 2,0.5 \%$ Triton X-100), with mouse IgG (CST, USA) as a control. The mixture was incubated with $20 \mu \mathrm{g}$ protein A/G agarose beads (GE, USA) at $4{ }^{\circ} \mathrm{C}$ overnight. The beads were washed three times with IP buffer and treated with $20 \mathrm{ng}$ proteinase $\mathrm{K}$ at $45^{\circ} \mathrm{C}$ for $30 \mathrm{~min}$. The DNA was purified and applied by PCR or qPCR. The primers are listed in Table S3.

\section{Mass spectrum analysis}

To detect $\triangle$ SMARCA5, a defective protein of SMAR CA5, SDS-PAGE of the target protein molecule weight region was used to analyze the protein mass spectrum. In brief, MCF-7 cells overexpressing circSMARCA5 were treated with $30 \mu \mathrm{M}$ MG132 for $24 \mathrm{~h}$. The cells were harvested, and the total protein was extracted for SDSPAGE. When the $90 \mathrm{kd}$ band and $110 \mathrm{kD}$ protein marker band were sufficiently separated, the electrophoresis was stopped, and the SDS-PAGE gel was excised between 70 and $90 \mathrm{kD}$ for analysis by mass spectrometry. Mass spectrometry data were analyzed by Mascot 2.3 and aligned with UniProt human_20190102_ 177661.fasta protein sequence data.

\section{Real-time quantitative PCR (qPCR)}

For RT-qPCR, total RNA was extracted from breast cancer tissues and adjacent tissues, rRNA was depleted, and RNase $\mathrm{R}$ was used to digest the linear RNA. qPCR was carried out as described [46]. In brief, total RNA was extracted by TRIzol reagent (Invitrogen, Carlsbad, CA), and CDNA was synthesized by random primers or oligodT (Yeasen ,China). qPCR was performed on a CFX 96 real-time PCR system using SYBR Green Real-time PCR Master Mix (Bio-Rad, USA). The expression levels of target genes were normalized by $2^{-\Delta \Delta C t}$ with GAPDH and $\beta$-actin as references. Each experiment was repeated three times. The primers used in $\mathrm{qPCR}$ are listed in Table S3.

\section{Single cell gel electrophoresis}

A comet assay was carried out as described [47]. In brief, $1 \times 10^{5}$ cells were mixed with $1 \mathrm{ml}$ agarose $(0.8 \%$ agarose in PBS). The suspension of agarose cells was dripped onto the slide to form a uniform film. The slide was 
incubated at $4{ }^{\circ} \mathrm{C}$ for $10 \mathrm{~min}$, and then placed in a slide box, followed by the addition of e precooled cracking liquid $(\mathrm{NaCl} 14.6 \mathrm{~g}, 100 \mathrm{~mm}$ EDTA $500 \mu \mathrm{l}, 0.4 \mathrm{M}$ Tris $250 \mu \mathrm{l}$, Triton $\mathrm{X} 100 \mathrm{ml}$, DMSO $10 \mathrm{ml}$, adjust solution $\mathrm{pH}$ to 10 with $\mathrm{NaOH}$ ). Avoiding light and using a constant $20 \mathrm{~V}$ voltage, electrophoresis was performed for 30 min. The slide was removed after electrophoresis and soaked 2 times in $0.4 \mathrm{~m}$ Tris solution (formula $9.69 \mathrm{~g}$ Tris with constant volume to $200 \mathrm{ml}$, and the solution $\mathrm{pH}$ was adjusted to $7.5 \mathrm{ml}$ ) for $5 \mathrm{~min}$ each. Then, the slides were removed and stained in $2 \mu \mathrm{g} / \mathrm{ml} \mathrm{EB}$ or acridine orange solution for $5 \mathrm{~min}$, washed twice with pure water for $10 \mathrm{~min}$, and observed under a fluorescence microscope. The cell trailing rate and mean tail length were analyzed and calculated by CASP software.

\section{Xenografts in nude mice}

Female BALB/cnu/nu nude mice, 28-30 days old and weighing $16-18 \mathrm{~g}$, were maintained under sterile conditions and fed sterile feed and water. The nude mice were divided into 4 groups, with 6 mice in each group. Each mouse was injected subcutaneously with prepared cells $\left(1 \times 10^{7}\right)$ at a single site. The tumor volume was measured with calipers and calculated using the formula VT $=1$ / $2(\mathrm{~L} X \mathrm{~W} \times \mathrm{W})$ (L: the maximum of tumor; W: the minimum length of tumor). When the tumor volume was 0.1 $\mathrm{cm}^{3}$, two groups were injected with cisplatin $(10 \mathrm{mg} / \mathrm{kg} /$ day), and the other two groups were given $1 \mathrm{X}$ PBS as a placebo. When the tumor of the control group grew to $200 \mu \mathrm{l}$, all mice were sacrificed, and the xenograft was removed, weighed and measured to determine tumor volume. All operations are carried out following the Guidelines for Animal Experimentation of Wuhan University. Our protocol was approved by the Ethics Committee for Animal Experimentation and was performed on the basis of the Guidelines for Animal Experimentation of Wuhan University and the National Institute of Health Guide for the Care and Use of Laboratory Animals.

\section{Immunohistochemistry}

Tissue sections $(5 \mu \mathrm{m})$ were deparaffinized in a gradient dilution of xylene and then hydrated in a gradient dilution of absolute ethanol. Subsequently, endogenous peroxidase activity was blocked by the freshly prepared solution of $3 \% \mathrm{H}_{2} \mathrm{O}_{2}$ for $10 \mathrm{~min}$ at room temperature. After antigen retrieval was performed in $0.02 \mathrm{M}$ PBS buffer ( $\mathrm{pH} 7.2-7.6$ ), 5\% BSA blocking was conducted at $37^{\circ} \mathrm{C}$ for $30 \mathrm{~min}$. After washing with PBS, the primary antibodies (Including anti-Smarca5, diluted 1:200, R; anti- $\gamma \mathrm{H} 2 \mathrm{AX}$, diluted $1: 100, \mathrm{R})$ were incubated at $4{ }^{\circ} \mathrm{C}$ overnight. The secondary antibody (diluted 1:1000, R) was incubated for $2 \mathrm{~h}$ at room temperature. Finally, the slides were stained with $\mathrm{DAB}$ and counterstained with hematoxylin. A Panoramic MIDI automatic digital slide scanner (3DHISTECH Ltd., Budapest, Hungary) was used for image processing and quantification. The expression levels of the target proteins (Smarca5, $\gamma \mathrm{H} 2 \mathrm{AX}$ ) in each tissue sample were examined based on the intensity of immunohistochemical staining.

\section{Flow cytometry}

The cell cycle was analyzed by flow cytometry. The Ex13-14, Ex15-16, and Ex13-16 sequences were inserted into a series of sequences of PCMV-DsRedT2A-MSC-EGFP from the MSC site in the form of recombinant vectors. After $48 \mathrm{~h}$ of transfection in the experimental group, the three recombinant vectors were stably transfected into a cell line expressing circSMARCA5 and a control cell line. The cells were harvested with trypsin, fully resuspended in a single cell suspension, and the negative control tubes and specimen tubes were sequenced according to the order of the samples, and the fluorescence was detected by flow cytometry. The two detection channels used were FITC-A and PE- A, respectively. For cell cycle, the cells were stained with PI Cell cycle kit (Yeasen, China) and detected by PE channel, which has the same excitation wavelength with PI. For the BrdU staining, 107 cells were labeled with $10 \mu \mathrm{M} \mathrm{BrdU}$ for $2 \mathrm{~h}$ at $37^{\circ} \mathrm{C}$. Cells were fixed and intracellularly stain with Anti-BrdU-APC antibody. The cells were counterstained with PI, and the stained cells were analyzed by flow cytometry. A total of 10,000 cells were detected, and the number of cells in each fluorescent region was analyzed. For cell transcription analysis, cells transfected with pDsRed-C1 or pEGFP-C1 (Addgene, USA) were used as channel gates to analyze the proportion of cells in each region.

\section{Chromatin Immunoprecipitation (ChIP)}

ChIP was carried out as described [48]. In brief, the cells were crosslinked with formaldehyde at a final concentration of $1 \%$ for $10 \mathrm{~min}$, and then glycine was added to a final concentration of $125 \mathrm{mM}$ and incubated with shaking for $5 \mathrm{~min}$. The cells were resuspended in cell lysis buffer (5 mM PIPES (pH 8.0), $85 \mathrm{mM} \mathrm{KCl}, 0.5 \%$ Nonidet $\mathrm{P}-40$ ) and incubated on ice for $10 \mathrm{~min}$ to allow the release of nuclei. The nuclear pellet was resuspended in MNase digestion buffer (0.32 M sucrose, $50 \mathrm{mM}$ Tris- $\mathrm{Cl}$ (pH 7.6), $4 \mathrm{mM} \mathrm{MgCl}_{2}, 1 \mathrm{mM} \mathrm{CaCl}, 0.1 \mathrm{mM}$ PMSF) for $1 \mathrm{~h}$ on ice. EDTA was added to $50 \mathrm{mM}$ to stop the digestion. The supernatant was then sonicated. After measuring the DNA concentration, fragmented chromatin (300-700 bp) was diluted with dilution buffer $(10 \mathrm{mM}$ Tris-Cl (pH 8.0), 0.5 mM EGTA, 1\% Triton X-100, 140 $\mathrm{mM} \mathrm{NaCl}$ ) supplemented with $1 \mathrm{mM}$ PMSF. The cleared supernatant was incubated with $2 \mu \mathrm{g}$ of rabbit anti-Rpb1 antibody (RNA polymerase II, CST, Danvers, MA, USA) 
or $2 \mu \mathrm{g}$ of anti-rabbit IgG (CST, Danvers, MA, USA) on a rocker overnight. After adding $40 \mu \mathrm{L}$ protein A/G Dyna-beads (Life Technologies, Carlsbad, CA), the reactions were incubated for $2 \mathrm{~h}$ at $4{ }^{\circ} \mathrm{C}$. The beads were washed with cell lysis buffer, low-salt wash buffer (200 $\mathrm{mM} \mathrm{NaCl}, 50 \mathrm{mM}$ Tris- $\mathrm{HCl}$ (pH 8.0), $5 \mathrm{mM} \mathrm{MgCl}_{2}, 1 \%$ Triton X-100), high-salt wash buffer (50 mM HEPES (pH 7.9), $500 \mathrm{mM} \mathrm{NaCl}, 1 \mathrm{mM}$ EDTA, $0.1 \%$ SDS, $1 \%$ Triton $\mathrm{X}$ 100, $0.1 \%$ deoxycholate), $\mathrm{LiCl}$ buffer $(250 \mathrm{mM} \mathrm{LiCl}, 100$ $\mathrm{mM}$ Tris- $\mathrm{HCl}$ (pH 8.0), $5 \mathrm{mM}$ EDTA, $0.5 \%$ Nadeoxycholate, $0.5 \%$ Triton X-100) and TE buffer $(100 \mathrm{mM}$ Tris- $\mathrm{Cl}$ (pH 7.5). The bound DNA was eluted and reversecrosslinked using elution buffer $(50 \mathrm{mM}$ Tris- $\mathrm{Cl}$ ( $\mathrm{pH} 8.0)$, $10 \mathrm{mM}$ EDTA, $1 \%$ SDS, $20 \mathrm{ng}$ proteinase $\mathrm{K}$ ) at $65^{\circ} \mathrm{C}$ overnight. The DNA was purified and analyzed by real-time qPCR assay. The specific primers (Table S3) were applied.

\section{Cycloheximide chase assay}

The cycloheximide assay was carried out as described [49]. MCF-7 cells were cultured in 6-well plates at a density of $1 \times 10^{5}$ cells/well and then transfected with plasmid pFLAG-CMV-SMARCA5 or FLAG-CMV- $\triangle$ SMARCA5 during the logarithmic phase. Forty-eight hours after transfection, cycloheximide was added to the medium at a final concentration of $50 \mu \mathrm{g} / \mathrm{ml}$. MCF-7 cells were harvested after treatment with cycloheximide for $0.5,1,2$, 4, or $8 \mathrm{~h}$. A western blot assay was applied to detect the expression level of SMRCA5 or $\triangle$ SMARCA5. A mAb against flag (AB clonal, Cambridge, MA, USA, dilution 1:1000) was used to detect SMARCA5 expression, and the rAb SMARCA5-Nterminal region (ABclonal, USA, dilution 1:1000) was used to detect $\triangle$ SMARCA5 expression.

\section{Phosphorthioate modification of DNA}

To detect the target sequence of circSMARCA5 binding to exon 15-16 DNA, phosphorylated DNA sequences were applied. In brief, the DNA sequence 5'-ATTTGA AGCATTTCATCTTTCCCAATTTTGTT-3' was synthesized with phosphorthioate modification of three bases at the $5^{\prime}$ and $3^{\prime}$ ends and named ANT. The mutation of ANT was named ANT-mut, with cytosine converted to guanine. The ANT phosphorthioate-modified DNA was applied to a dot-blot assay at a concentration of $10 \mu \mathrm{g} / \mathrm{ml}$ to examine the binding ability to circSMARCA5. Then, 10 pmol of ANT phosphorthioatemodified DNA was transfected into MCF-7 cells in each well of a six-well plate for $48 \mathrm{~h}$, and the SMARCA5 protein expression level was detected by western blotting.

\section{Immunofluorescence}

The cells were inoculated on the slide and cultured in a 24well plate. After DNA damage drugs (cisplatin or bleomycin) were applied for $24 \mathrm{~h}$, the cells were washed with PBS. Subsequently, the cells were fixed with $4 \%$ paraformaldehyde and then permeabilized with $0.5 \%$ Triton X-100 in PBS for $10 \mathrm{~min}$ at room temperature. After treating the cells with $5 \% \mathrm{BSA}$ for $30 \mathrm{~min}$ at room temperature, the slides were incubated with antibody (anti-SMARCA 5 or anti- $\gamma \mathrm{H} 2 \mathrm{AX}$ ) overnight at $4{ }^{\circ} \mathrm{C}$. After washing with $1 \mathrm{X}$ PBST $(0.1 \%$ Tween 20 in PBS) 3 times, the cells were subjected to indirect immunofluorescence using a fluorescence-labeled antibody (rabbit 594/488, Proteintech, USA). The nuclei were stained with DAPI. The images were taken using a fluorescence microscope (Olympus, Japan).

\section{Nuclear and cytoplasmic separation}

The cells were collected and resuspended in cell lysate buffer (1\% NP40, $5 \mathrm{nM}$ EDTA, and 0.5\% sodium deoxycholate) for $5 \mathrm{~min}$, followed by centrifugation at 4000 $\mathrm{rpm}$ for $1 \mathrm{~min}$ at $4{ }^{\circ} \mathrm{C}$. The cytoplasmic proteins were present in the supernatant. The pellet was washed with cell lysate buffer for $10 \mathrm{~min}$ at $4{ }^{\circ} \mathrm{C}$ and then centrifuged for $5 \mathrm{~min}$ at $4{ }^{\circ} \mathrm{C}$ to collect the nuclei.

\section{Statistical analyses}

All statistical analysis was done with the software Graphpad Prism (GraphPad Software, La Jolla, CA) and $\mathrm{R}$ packages (www.r-project.org).

\section{Supplementary information}

Supplementary information accompanies this paper at https://doi.org/10. 1186/s12943-020-01246-X.

Additional file 1: Figure S1. The expression level of SMARCA5 and circSMARCA5 in blood and tisssues sample of breast cancer patients health volunteers and tisssues sample of renal cancer. Figure S2. RNA FISH showing circSMARCA5 was mainly expressed in the nucleus. Figure S3. circSMARCA5 decreases the expression of SMARCA5 in MCF-7 cells. Figure S4. The protein level of SMARCA5 in breast cancer and adjacent normal tissues. Figure S5. The expression correlation of circSMARCA5 and SMARCA5in different tumors. Figure S6. circSMARCA5 overexpression downregulated the protein levels of SMARCA5 while upregulating the truncated SMARCA5 ( $\triangle$ SMARCA5) protein levels. Figure S7. Fragmented genomic DNA for DRIP-qPCR experiments. Figure S8. The secondary structure of circSMARCA5 and circSMARCA5-mut. Figure S9. circSMARCA5 has no significant effect on the proliferation and migration ability of breast cancer cells. Figure S10. The truncated protein $\triangle S M A R C A 5$ is a nonfunctional protein product. Figure S11. Immunofluorescence assay using a $\mathrm{YH} 2 \mathrm{AX}$ antibody showing that the cotransfection of ANT in circSMARCA5-overexpressing cells can abrogate $\mathrm{YH} 2 \mathrm{AX}$ levels inducedby circSMARCA5 but the cotransfection of ANT-mut cannot. Figure S12. ANT significantly decreased the degree of colocalization between circSMARCA5 and its cognate DNA locus. Figure S13. (A) MCF-7 cells expressing control vector, circSMARCA5 or circSMARCA5-mut were treated with cisplatin or bleomycin in concentration gradient for $24 \mathrm{~h}$, and CCK8 was used to measure cell viability. Figure S14. circSMARCA5 downregulate SMARCA5 and suppress DNA damage repair in Hela cell.

Additional file 2: Table S1. The ratio of circ-to-linear of circSMARCA5 (expression of circRNA / linear host genes) and clinical pathologic characteristics of breast cancer.

Additional file 3: Table S2. Probes for circRNA detection.

Additional file 4: Table S3. Probe for transcribed RNA and expression plasmid. 


\section{Abbreviations}

circRNA: circular RNA; RBP: RNA binding protein; RNA-Seq: RNA sequencing; qPCR: Real-time quantitative polymerase chain reaction; HER2: Human epidermal growth factor receptor 2; ISH: In situ hybridization; IHC: Immunohistochemistry; IF: Immunofluorescence; RIP: RNA immunoprecipitation; ChIP: Chromatin Immunoprecipitation; DRIP: DNA-RNA immunoprecipitation; NB: Northern blot; FISH: Fluorescence In Situ Hybridization; 3'RACE: The 3' rapid amplification of cDNA ends; MS: Mass Spectrum

\section{Acknowledgements}

The authors would like to thank Lingling Chen in Shanghai Institute of Biochemistry and Cell Biology, Chinese Academy of Sciences for the technical support and manuscript discussion. The author would also like to thank Wuhan University for financial support to this research and the Nature Research Editing Service for language editing.

\section{Authors' contributions}

XX, ZJ and TY carried out the experiments, data collection and analysis. GY, DX and CW prepared the high throughput sequencing and carried out the data analysis. YX, YW and XJ carried out the result correction and integration. CK, HC and WL designed the work and wrote the manuscript. All authors read and approved the final manuscript.

\section{Funding}

This work was supported by the Science Fund for Creative Research Groups of the Natural Science Foundation of Hubei under Grant [zrqt2019000039] to L. W, the National Natural Science Foundation of China [81870129] to C. H, the China National Grand S\&T Special Project [2018ZX10733403] to C. H, and the Medical Science Advancement Program (Basic Medical Science) of Wuhan University [TFJC2018003] to C. H and the National Natural Science Foundation of China [81772721] to K. C.

\section{Availability of data and materials}

All RNASeq raw data can be accessed in the NCBI GEO database (https:// www.ncbi.nlm.nih.gov/geo/query/acc.cgi?acc=GSE133998, accession code: wxmjuumulvsbbuf).

\section{Ethics approval and consent to participate}

The study was approved by the Ethics Committee (Institutional Review Board) of Wuhan University Zhongnan Hospital. All patients provided written informed consent for participation according to the Declaration of Helsinki and written informed consent for publication. The clinical information was retrieved from the medical records. All in vivo operations were carried out following the Guidelines for Animal Experimentation of Wuhan University. Our protocol was approved by the Ethics Committee for Animal Experimentation and was performed on the basis of the Guidelines for Animal Experimentation of Wuhan University and the National Institute of Health Guide for the Care and Use of Laboratory Animals.

\section{Consent for publication}

Not applicable.

\section{Competing interests}

The authors declare that they have no competing interests exist.

\section{Author details}

${ }^{1}$ School of Basic Medical Sciences, Wuhan University, Wuhan 430071, Hubei, China. ${ }^{2}$ Hubei Key Laboratory of Tumor Biological Behaviors, Department of Breast and Thyroid Surgery, Hubei Cancer Clinical Study Center, Zhongnan Hospital, Wuhan University, Wuhan 430071, Hubei, China. ${ }^{3}$ Department of Urology, Tongji Hospital, Tongji Medical College, Huazhong University of Science and Technology, Wuhan 430030, China. ${ }^{4}$ College of Biomedicine and Health, Huazhong Agricultural University, Wuhan 430070, China. ${ }^{5}$ Hubei Province Key Laboratory of Allergy and Immunology, Wuhan 430071, Hubei, China.
Received: 27 April 2020 Accepted: 12 August 2020

Published online: 24 August 2020

\section{References}

1. Chen LL. The biogenesis and emerging roles of circular RNAs. Nat Rev Mol Cell Biol. 2016;17:205-11.

2. Jeck WR, Sharpless NE. Detecting and characterizing circular RNAs. Nat Biotechnol. 2014;32:453-61.

3. Arnaiz E, Sole C, Manterola L, Iparraguirre L, Otaegui D, Lawrie CH. CircRNAs and cancer: Biomarkers and master regulators. Semin Cancer Biol. 2019;58: 90-99.

4. Chen J, Li Y, Zheng Q, Bao C, He J, Chen B, Lyu D, Zheng B, Xu Y, Long Z, et al. Circular RNA profile identifies circPVT1 as a proliferative factor and prognostic marker in gastric cancer. Cancer Lett. 2017;388:208-19..

5. Li P, Chen H, Chen S, Mo X, Li T, Xiao B, Yu R, Guo J. Circular RNA 0000096 affects cell growth and migration in gastric cancer. Br J Cancer. 2017;116: 626-33.

6. Zhang Y, Liu H, Li W, Yu J, Li J, Shen Z, Ye G, Qi X, Li G. CircRNA_100269 is downregulated in gastric cancer and suppresses tumor cell growth by targeting miR-630. Aging (Albany NY). 2017;9:1585-94.

7. Zhang J, Liu H, Hou L, Wang G, Zhang R, Huang Y, Chen X, Zhu J. Circular RNA_LARP4 inhibits cell proliferation and invasion of gastric cancer by sponging miR-424-5p and regulating LATS1 expression. Mol Cancer. 2017; 16:151.

8. Weng W, Wei Q, Toden S, Yoshida K, Nagasaka T, Fujiwara T, Cai S, Qin H, Ma Y, Goel A. Circular RNA ciRS-7-a promising prognostic biomarker and a potential therapeutic target in colorectal Cancer. Clin Cancer Res. 2017;23: 3918-28.

9. Yu L, Gong X, Sun L, Zhou Q, Lu B, Zhu L. The circular RNA Cdr1as act as an oncogene in hepatocellular carcinoma through targeting miR-7 expression. PLoS One. 2016;11:e0158347.

10. Tang H, Huang X, Wang J, Yang L, Kong Y, Gao G, Zhang L, Chen ZS, Xie X. circKIF4A acts as a prognostic factor and mediator to regulate the progression of triple-negative breast cancer. Mol Cancer. 2019;18:23.

11. Sang Y, Chen B, Song X, Li Y, Liang Y, Han D, Zhang N, Zhang H, Liu Y, Chen T, et al. circRNA_0025202 Regulates Tamoxifen Sensitivity and Tumor Progression via Regulating the miR-182-5p/FOXO3a Axis in Breast Cancer. Mol Ther. 2019;27:1638-52.

12. Fu B, Zhang A, Li M, Pan L, Tang W, An M, Liu W, Zhang J. Circular RNA profile of breast cancer brain metastasis: identification of potential biomarkers and therapeutic targets. Epigenomics. 2018;10:1619-30.

13. Zhong Y, Du Y, Yang X, Mo Y, Fan C, Xiong F, Ren D, Ye X, Li C, Wang Y, et al. Circular RNAs function as ceRNAs to regulate and control human cancer progression. Mol Cancer. 2018;17:79.

14. Du WW, Zhang C, Yang W, Yong T, Awan FM, Yang BB. Identifying and characterizing circRNA-protein interaction. Theranostics. 2017;7:4183-91.

15. Legnini I, Di Timoteo G, Rossi F, Morlando M, Briganti F, Sthandier O, Fatica A, Santini T, Andronache A, Wade M, et al. Circ-ZNF609 is a circular RNA that can be translated and functions in Myogenesis. Mol Cell. 2017;66:22-37 e29.

16. Pamudurti NR, Bartok $O$, Jens M, Ashwal-Fluss R, Stottmeister $C$, Ruhe $L$, Hanan M, Wyler E, Perez-Hernandez D, Ramberger E, et al. Translation of CircRNAs. Mol Cell. 2017;66:9-21 e27.

17. Conn VM, Hugouvieux V, Nayak A, Conos SA, Capovilla G, Cildir G, Jourdain A, Tergaonkar V, Schmid M, Zubieta C, Conn SJ. A circRNA from SEPALLAT $A 3$ regulates splicing of its cognate mRNA through R-loop formation. Nat Plants. 2017;3:17053.

18. Aydin OZ, Vermeulen W, Lans H. ISWI chromatin remodeling complexes in the DNA damage response. Cell Cycle. 2014;13:3016-25.

19. Dirscherl SS, Krebs JE. Functional diversity of ISWI complexes. Biochem Cell Biol. 2004;82:482-9.

20. Erdel F, Rippe K. Binding kinetics of human ISWI chromatin-remodelers to DNA repair sites elucidate their target location mechanism. Nucleus. 2011;2: 105-12.

21. Broering TJ, Wang YL, Pandey RN, Hegde RS, Wang SC, Namekawa SH. BAZ1B is dispensable for H2AX phosphorylation on tyrosine 142 during spermatogenesis. Biol Open. 2015;4:873-84.

22. Toiber D, Erdel F, Bouazoune K, Silberman DM, Zhong L, Mulligan P, Sebastian C, Cosentino C, Martinez-Pastor B, Giacosa S, et al. SIRT6 recruits SNF2H to DNA break sites, preventing genomic instability through chromatin remodeling. Mol Cell. 2013;51:454-68. 
23. Mueller AC, Sun D, Dutta A. The miR-99 family regulates the DNA damage response through its target SNF2H. Oncogene. 2013;32:1164-72.

24. Gigek CO, Lisboa LC, Leal MF, Silva PN, Lima EM, Khayat AS, Assumpcao PP, Burbano RR, Smith Mde A. SMARCA5 methylation and expression in gastric cancer. Cancer Investig. 2011;29:162-6.

25. Yu J, Xu QG, Wang ZG, Yang Y, Zhang L, Ma JZ, Sun SH, Yang F, Zhou WP. Circular RNA cSMARCA5 inhibits growth and metastasis in hepatocellular carcinoma. J Hepatol. 2018;68:1214-27.

26. Tian JDC, Liang L. Involvement of circular RNA SMARCA5/microRNA-620 axis in the regulation of cervical cancer cell proliferation, invasion and migration. Eur Rev Med Pharmacol Sci. 2018;22:8589-98.

27. Liu H, Wu Y, Wang S, Jiang J, Zhang C, Jiang Y, Wang X, Hong L, Huang H. Circ-SMARCA5 suppresses progression of multiple myeloma by targeting miR-767-5p. BMC Cancer. 2019;19:937.

28. Wang Y, Li H, Lu H, Qin Y. Circular RNA SMARCA5 inhibits the proliferation migration, and invasion of non-small cell lung cancer by miR-19b-3p/ HOXA9 axis. Onco Targets Ther. 2019:12:7055-65.

29. Mathews DH, Disney MD, Childs JL, Schroeder SJ, Zuker M, Turner DH. Incorporating chemical modification constraints into a dynamic programming algorithm for prediction of RNA secondary structure. Proc Natl Acad Sci U S A. 2004;101:7287-92.

30. Kubota Y, Shimizu S, Yasuhira S, Horiuchi S. SNF2H interacts with XRCC1 and is involved in repair of $\mathrm{H}_{2} \mathrm{O} 2$-induced DNA damage. DNA Repair (Amst). 2016;43:69-77.

31. Lan L, Ui A, Nakajima S, Hatakeyama K, Hoshi M, Watanabe R, Janicki SM, Ogiwara H, Kohno T, Kanno S, Yasui A. The ACF1 complex is required for DNA double-strand break repair in human cells. Mol Cell. 2010;40:976-87.

32. Jin Q, Mao X, Li B, Guan S, Yao F, Jin F. Overexpression of SMARCA5 correlates with cell proliferation and migration in breast cancer. Tumour Biol. 2015:36:1895-902.

33. Yang Z, Xie L, Han L, Qu X, Yang Y, Zhang Y, He Z, Wang Y, Li J. Circular RNAs: regulators of Cancer-related signaling pathways and potential diagnostic biomarkers for human cancers. Theranostics. 2017;7:3106-17.

34. Wang Y, Mo Y, Gong Z, Yang X, Yang M, Zhang S, Xiong F, Xiang B, Zhou M, Liao Q, et al. Circular RNAs in human cancer. Mol Cancer. 2017;16:25.

35. Dong Y, He D, Peng Z, Peng W, Shi W, Wang J, Li B, Zhang C, Duan C. Circular RNAs in cancer: an emerging key player. J Hematol Oncol. 2017;10:2.

36. Ponde NF, Zardavas D, Piccart M. Progress in adjuvant systemic therapy for breast cancer. Nat Rev Clin Oncol. 2019;16:27-44.

37. Harbeck N, Gnant M. Breast cancer. Lancet. 2017;389:1134-50.

38. Munzone E, Colleoni M. Clinical overview of metronomic chemotherapy in breast cancer. Nat Rev Clin Oncol. 2015;12:631-44.

39. Li Z, Huang C, Bao C, Chen L, Lin M, Wang X, Zhong G, Yu B, Hu W, Dai L, et al. Exon-intron circular RNAs regulate transcription in the nucleus. Nat Struct Mol Biol. 2015;22:256-64.

40. Li X, Liu CX, Xue W, Zhang Y, Jiang S, Yin QF, Wei J, Yao RW, Yang L, Chen LL. Coordinated circRNA biogenesis and function with NF90/NF110 in viral infection. Mol Cell. 2017;67:214-27 e217.

41. Cui X, Wang J, Guo Z, Li M, Li M, Liu S, Liu H, Li W, Yin X, Tao J, Xu W. Emerging function and potential diagnostic value of circular RNAs in cancer. Mol Cancer. 2018;17:123.

42. Barrett SP, Salzman J. Circular RNAs: analysis, expression and potential functions. Development. 2016:143:1838-47.

43. Ma XK, Wang MR, Liu CX, Dong R, Carmichael GG, Chen LL, Yang L. CIRCexplorer3: a CLEAR pipeline for direct comparison of circular and linear RNA expression. Genomics Proteomics Bioinformatics. 2019;17:511-21.

44. Powell WT, Coulson RL, Gonzales ML, Crary FK, Wong SS, Adams S, Ach RA, Tsang P, Yamada NA, Yasui DH, et al. R-loop formation at Snord116 mediates topotecan inhibition of Ube3a-antisense and allele-specific chromatin decondensation. Proc Natl Acad Sci U S A. 2013;110:13938-43.

45. Ginno PA, Lott PL, Christensen HC, Korf I, Chedin F. R-loop formation is a distinctive characteristic of unmethylated human $\mathrm{CpG}$ island promoters. Mol Cell. 2012;45:814-25

46. Livak KJ, Schmittgen TD. Analysis of relative gene expression data using real-time quantitative $P C R$ and the 2(-Delta Delta $C(T))$ method. Methods. 2001:25:402-8

47. Kiziltepe T, Hideshima T, Ishitsuka K, Ocio EM, Raje N, Catley L, Li CQ, Trudel $\mathrm{L}$, Yasui $\mathrm{H}$, Vallet $\mathrm{S}$, et al. JS-K, a GST-activated nitric oxide generator, induces DNA double-strand breaks, activates DNA damage response pathways, and induces apoptosis in vitro and in vivo in human multiple myeloma cells. Blood. 2007;110:709-18.
48. Kim TH, Dekker J. ChIP-Quantitative Polymerase Chain Reaction (ChIP-qPCR). Cold Spring Harb Protoc. 2018;2018. https://doi.org/10.1101/pdb.prot082628.

49. Majumder P, Chen YT, Bose JK, Wu CC, Cheng WC, Cheng SJ, Fang YH, Chen YL, Tsai KJ, Lien CC, Shen CK. TDP-43 rsssegulates the mammalian spinogenesis through translational repression of Rac1. Acta Neuropathol. 2012;124:231-45.

\section{Publisher's Note}

Springer Nature remains neutral with regard to jurisdictional claims in published maps and institutional affiliations.
Ready to submit your research? Choose BMC and benefit from:

- fast, convenient online submission

- thorough peer review by experienced researchers in your field

- rapid publication on acceptance

- support for research data, including large and complex data types

- gold Open Access which fosters wider collaboration and increased citations

- maximum visibility for your research: over $100 \mathrm{M}$ website views per year

At BMC, research is always in progress.

Learn more biomedcentral.com/submissions 